\title{
Error Probability Analysis and Power Allocation for Interference Exploitation Over Rayleigh Fading Channels
}

\author{
Abdelhamid Salem, Member, IEEE, and Christos Masouros, Senior Member, IEEE,
}

\begin{abstract}
This paper considers the performance analysis of constructive interference (CI) precoding technique in multiuser multiple-input single-output (MU-MISO) systems with a finite constellation phase-shift keying (PSK) input alphabet. Firstly, analytical expressions for the moment generating function (MGF) and the average of the received signal-to-noiseratio (SNR) are derived. Then, based on the derived MGF expression the average symbol error probability (SEP) for the CI precoder with PSK signaling is calculated. In this regard, new exact and very accurate asymptotic approximation for the average SEP are provided. Building on the new performance analysis, different power allocation schemes are considered to enhance the achieved SEP. In the first scheme, power allocation based on minimizing the sum symbol error probabilities (Min-Sum) is studied, while in the second scheme the power allocation based on minimizing the maximum SEP (Min-Max) is investigated. Furthermore, new analytical expressions of the throughput and power efficiency of the CI precoding in MUMISO systems are also derived. The numerical results in this work demonstrate that, the CI precoding outperforms the conventional interference suppression precoding techniques with an up to $20 \mathrm{~dB}$ gain in the transmit SNR in terms of SEP, and up to $15 \mathrm{~dB}$ gain in the transmit SNR in terms of the throughput. In addition, the SEP-based power allocation schemes provide additional up to $13 \mathrm{~dB}$ gains in the transmit SNR compared to the conventional equal power allocation scheme.
\end{abstract}

Index Terms-Multi-user MISO, interference exploitation, phase-shift keying signaling, SEP.

\section{INTRODUCTION}

Multi-user multiple-input single-output (MU-MISO) communication system has been recognized as a promising technique in wireless communication networks [2]-[4]. However, the performance of MU-MISO systems can be impacted by multi-user interferences in practical applications [2]-[4]. Consequently, a large number of researches have considered the impact of the interference in $\mathrm{MU}$ MISO systems, and several techniques have been introduced to mitigate the multi-user interference in MU-MISO channels [4]-[6]. For instance, in the applications when the channel state information (CSI) is perfectly known at the base station (BS), dirty-paper coding (DPC) technique has been proposed [7]-[10]. In DPC technique the channel

The authors are with the department of Electronic and Electrical Engineering, University College London, London, UK, (emails: \{a.salem, c.masouros\}@ucl.ac.uk).

Part of this paper has been accepted in WCNC 2020 [1]. capacity is achieved by removing the interference before the transmission. However, DPC is difficult if not impossible to implement in practical communication networks, due to its very high complexity [7]-[10]. Therefore, low complexity linear precoding techniques, such as zero-forcing (ZF), have received significant research interest [11], [12]. Furthermore, precoding techniques based on optimization have also widely studied and investigated in literature [13][16]. In this regard, several optimization-based schemes have been proposed in different areas. For instance, signalto-interference-plus-noise ratio (SINR) balancing technique is a precoding scheme that depends on maximizing the minimum SINR subject to different transmission power constraints [13], [14]. In addition, minimizing the transmission power precoding is another precoding scheme that aims to minimize the transmission power subject to a minimum threshold value of the SINR [15], [16].

However, all the above precoding/transmission schemes have ignored the fact that the interference in communication systems can be beneficial to the received signal, and thus the inherent interference in MU-MISO systems can be exploited to further enhance its performance. In light of this, constructive interference $(\mathrm{CI})$ precoding technique has received significant research interest in the past few years [17]-[22]. The main idea of the CI precoding scheme is to exploit the interference that can be known to the $\mathrm{BS} /$ transmitter to enhance the system performance. That is, with the knowledge of both the users' channels and data symbols, the BS can classify the interference as constructive and destructive. The constructive interference is the interference that can push the received symbol deeper in the constructive/detection region of the constellation point of interest. According to this methodology, the preceder can be designed to make all the multi-user interferences constructive to the desired symbol. For clarity, the basic concept of CI precoding is summarized in Fig. 1 for QPSK constellation. In brief, the constructive interference moves the received symbol away from the decision thresholds of the constellation, this represents the green areas in the constellation of Fig. 1, and thus enhances the detection. For more details, we refer the reader to [17] where the CI precoding technique was discussed and the application of $\mathrm{CI}$ in different wireless communication networks was investigated. 


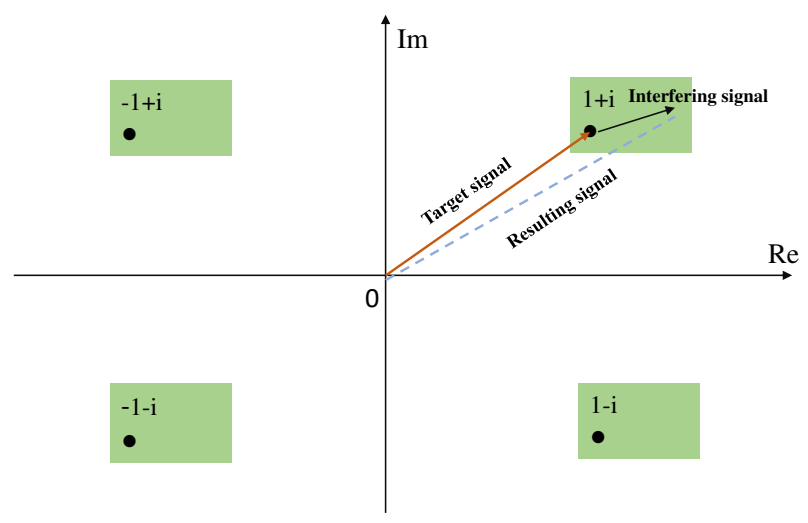

Figure 1: Constructive interference in QPSK, where the constructive regions are denoted by the green areas.

The concept of interference exploitation technique has been widely considered in literature [17]-[20], [22]. This line of research was introduced in [18], where the CI precoding technique has been proposed for downlink MIMO systems. The system performance in this work has been enhanced by exploiting some of the existent inter-channel interference (ICI). This exploitation was performed by applying partial channel inversion such that the constructive part of ICI is preserved and exploited while the destructive part is eliminated. As a result the effective SINR has been improved without increasing the BS transmission power. In [19], [20], a data-aided transmit precoding technique for downlink MU-MISO system was proposed. The authors in this work used the knowledge of the data and channel state information (CSI) to exploit the constructive interference in the system. The proposed precoder reduces the transmit power compared to the conventional schemes, by adapting the quality of service (QoS) constraints to accommodate CI as a source of useful signal power. In [22] a low-complexity vector precoding scheme for downlink MU-MISO system with limited feedback was proposed. More specifically, the authors proposed a modified vector-perturbation (VP) technique where the search of perturbing vectors is limited to the area where the distances from the decision thresholds are increased with respect to a distance threshold. By implementing this, the perturbation quantities can improve the detection and no need to be removed at the users. Further works in [23], [24] considered general category of CI regions, and several properties for this region have been presented and discussed. Furthermore, recently closedform expression for CI precoding technique in MU-MISO systems with PSK signaling has been derived in [10]. This expression has designed based on optimal performance, thus its performance is equivalent to the CI schemes considered in literature, for instance [19], [22]. The CI closed-form expression has paved the way to develop theoretical analysis of the CI technique. Based on this precoding expression, in our previous works in [25]-[28] closed-form expressions of the achievable sum-rate of the CI precoding technique in
MU-MISO systems under PSK signaling have been derived and investigated.

Accordingly in this paper we analyze the performance of CI precoding in MU-MISO systems with $M$-PSK signals. In this regard, the average symbol error probability (SEP) of the CI precoding is analyzed. Then, two different power allocation schemes to minimize the sum symbol error probabilities (Min-Sum), and to minimize the maximum average SEP (Min-Max) are considered. Furthermore, the throughput and power efficiency achieved by the CI precoding in MU-MISO systems are also studied.

For clarity, the major contributions of this work are listed below.

1) Mathematical expressions for the MGF and the average of the received SNR for CI precoding technique under $M$-PSK inputs are derived.

2) Analytical expression of the average SEP for CI precoding with $M$-PSK is derived. For simplicity and mathematical tractability, new and very accurate asymptotic approximation of the SEP is also provided.

3) Two power allocation schemes to improve the SEP and enhance the system performance are proposed. In the first one, we consider power allocation technique that aims to minimize the sum symbol error probabilities subject to total power constraint. Whilst in the second scheme, we study power allocation technique that aims to minimize the maximum SEP subject to total power constraint.

4) Based on the above analysis, closed form expression of the power allocation factors are presented.

5) Analytical expressions for the throughput and power efficiency for the CI precoding in MU-MISO systems under $M$-PSK inputs are also derived.

The numerical results in this paper show that, for a given SEP the CI precoding can provide up to $20 \mathrm{~dB}$ gain in the transmit SNR compared to the conventional interference suppression precoding techniques. In addition, increasing the transmit SNR, number of users and number of BS antennas always enhance the achieved SEP. Furthermore, by using the derived analysis specifically tailored power allocation schemes provide additional up to $13 \mathrm{~dB}$ gains in the transmit SNR compared to the conventional transmission scheme. Finally, the CI precoding outperforms the conventional interference suppression precoding technique in terms of throughput for a wide range with an up to 15 $\mathrm{dB}$ gain in the transmit SNR.

Next, Section II describes the MU-MISO system model. Section III, derives the analytical expressions for the moment generating function and the average received SNR. Section IV derives the exact and approximated analytical expressions for the average symbol error probability. Section V, considers symbol error minimization through different power allocation schemes, minimizing the sum symbol error probabilities and minimizing the maximum symbol error probability. Section VI, considers the throughput and power efficiency for the CI precoding in MU- 
MISO systems. The graphical illustrations of the results are presented and discussed in Section VII. Finally, our conclusions are presented in Section VIII.

\section{SySTEM MODEL}

We consider a down-link MU-MISO system, consisting of $N$-antennas BS communicating simultaneously with $K$ single antenna users. The $K \times N$ channel matrix between the BS and the $K$ users is denoted by $\mathbf{H}$, which can be expressed as $\mathbf{H}=\mathbf{D}^{1 / 2} \tilde{\mathbf{H}}$ where the $K \times N$ matrix $\tilde{\mathbf{H}}$ models the small-scale fading coefficients which are modeled as independent, circularly symmetric complex Gaussian random variables with mean zero and variance one, and $\mathbf{D}$ is a $K \times K$ diagonal matrix in which $[\mathbf{D}]_{k k}=\varpi_{k}=d_{k}^{-m}$ where $d_{k}$ denotes the distance from the BS to the $k^{t h}$ user and $m$ denotes the path-loss exponent. It is also assumed that the CSI is perfectly known at the BS ${ }^{1}$. The received signal at the $k^{\text {th }}$ user in the considered system can be written as,

$$
y_{k}=\mathbf{h}_{k} \mathbf{W} \mathbf{x}+n_{k},
$$

where $\mathbf{x}$ is $K \times 1$ PSK-modulated signal vector, $\mathbf{W}$ is $N \times K$ precoding matrix, $\mathbf{h}_{k}$ is the $1 \times N$ channel vector of user $k$, and $n_{k}$ is the additive wight Gaussian noise (AWGN) at the $k^{t h}$ user, $n_{k} \sim \mathcal{C N}\left(0, \sigma_{k}^{2}\right)$. The closed-form expression for CI precoding with PSK signaling ${ }^{2}$ can be expressed as [10], [25], [26]

$$
\mathbf{W}=\frac{1}{K} \beta \mathbf{H}^{H}\left(\mathbf{H H}^{H}\right)^{-1} \operatorname{diag}\left\{\mathbf{V}^{-1} \mathbf{u}\right\} \mathbf{x x}^{H},
$$

where $\beta=\sqrt{P_{p}} \beta_{p}, P_{p}$ is the total transmit power, $\beta_{p}$ is the scaling factor, $\beta_{p}=\sqrt{\frac{1}{\mathbf{u}^{H} \mathbf{V}^{-1} \mathbf{u}}}, \mathbf{V}$ is a $K \times K$ matrix and given by $\mathbf{V}=\operatorname{diag}\left(\mathbf{x}^{H}\right)\left(\mathbf{H H}^{H}\right)^{-1} \operatorname{diag}(\mathbf{x})$ and $\mathbf{u}$ is a $K \times 1$ vector $\mathbf{1}^{T} \mathbf{u}=1^{3}$. As by CI precoding the resulting interference contributes to the useful signal power, it has been shown in [18], [19] that the the instantaneous received SNR at user $k$ using CI precoding technique can be written as ${ }^{4}$

\footnotetext{
${ }^{1}$ In this work, for simplicity and to gain an understanding of using $\mathrm{CI}$ to enhance the performance of MU-MISO systems, we assume that there is no correlation between the transmit antennas at the BS. The issue of correlated channels will be investigated in future work.

${ }^{2}$ Please note that, CI precoding is most useful in high interference scenarios with low SINR, where there is significant interference to exploit. In such scenarios low order modulations such as BPSK and QPSK are typically used to secure reliable performance. Accordingly, the analysis for PSK signals is more topical for the practical finite constellation scenarios of this paper.

${ }^{3}$ Please, note that CI precoding expression is conditioning on $\mathbf{u}$, thus all the derived expressions are conditioning on $\mathbf{u}$. The values of this vector should satisfy the condition $\mathbf{1}^{T} \mathbf{u}=1$. The impact of this vector on the system performance has been investigated in [10], [25]. Although the elements of $\mathbf{u}$ should be selected to maximize the performance, using any values larger than zero and satisfy $\mathbf{1}^{T} \mathbf{u}=1$ can show the superiority of the CI over the conventional techniques.

${ }^{4}$ For more details we refer the reader to [18], [19].
}

$$
\gamma_{k}=\frac{\left|\boldsymbol{h}_{k} \mathbf{W} \mathbf{x}\right|^{2}}{\sigma_{k}^{2}} .
$$

In the following sections we will study the statistics of the received SNR and analyze the performance of CI precoding technique in details.

\section{MGF AND AVERAGE SNR FOR CI PRECODING}

In this section, we derive the MGF and the average SNR expressions of the considered MU-MISO system. To start with, by substituting (2) into (3), the SNR at user $k$ using CI precoding technique can be expressed as

$$
\gamma_{k}=\frac{\left|\boldsymbol{h}_{k} \frac{\sqrt{P_{p}} \beta_{p}}{K} \mathbf{H}^{H}\left(\mathbf{H} \mathbf{H}^{H}\right)^{-1} \operatorname{diag}\left\{\mathbf{V}^{-1} \mathbf{u}\right\} \mathbf{x}\right|^{2}}{\sigma_{k}^{2}} .
$$

For simplicity, the scaling factor $\beta_{p}$ is defind as, $\beta_{p}=$ $\frac{1}{\sqrt{\mathbf{u}^{H} \operatorname{diag}\left(\mathbf{x}^{H}\right)^{-1} N \mathbf{D}(\operatorname{diag}(\mathbf{x}))^{-1} \mathbf{u}}}$, [25], [26], [29]. The received SNR in (4) can be expressed also as

$$
\gamma_{k}=\frac{\left|\frac{\sqrt{P_{p}} \beta_{p}}{K} \mathbf{a}_{k} \mathbf{A} \mathbf{u} x_{k}\right|^{2}}{\sigma_{k}^{2}},
$$

where $\mathbf{a}_{k}$ is a $1 \times K$ vector the $k^{t h}$ element of this vector is one and all the other elements are zeros, and $\mathbf{A}=\mathbf{V}^{-1}$. We can re-write the SNR expression in (5) as

$$
\gamma_{k}=\frac{\left|\frac{\sqrt{P_{p}} \beta_{p}}{K} \mathbf{a}_{k} \mathbf{D} \mathbf{u} \frac{\mathbf{a}_{k} \mathbf{A u}}{\mathbf{a}_{\mathbf{k}} \mathbf{D u}} x_{k}\right|^{2}}{\sigma_{k}^{2}}=\alpha_{k}|g|^{2},
$$

where $\alpha_{k}=\frac{\left|\frac{\sqrt{P_{p}} \beta_{p}}{K} \mathbf{a}_{k} \mathbf{D u}\right|^{2}}{\sigma_{k}^{2}}$ and $g=\frac{\mathbf{a}_{k} \mathbf{A u}}{\mathbf{a}_{k} \mathbf{D u}}$. From the results in [30]-[33], $g$ has conditional Gamma distribution with shape parameter $\nu$ and scale parameter $\theta, g \sim \Gamma(\nu, \theta)$. The optimal performance of the $\mathrm{CI}$ is attained with $\nu=N$ and $\theta=1$. Consequently, the received SNR, $\gamma_{k}$, has General Gamma distribution, $\gamma_{k} \sim \Gamma(p, d, a)$, with $p=\frac{1}{2}, d=\frac{\nu}{2}$ and $a=\alpha_{k}$. Therefore, the cumulative distribution function (CDF) and the probability density function (PDF) of the received SNR, $\gamma_{k}$, can be written, respectively, as

$$
\begin{aligned}
F_{\gamma_{k}}(\gamma) & =\left(\frac{\varphi\left(d / p,(\gamma / a)^{p}\right)}{\Gamma(d / p)}\right), \\
\text { and } f_{\gamma_{k}}(\gamma) & =\left(\frac{\left(\frac{p}{a^{d}}\right) \gamma^{d-1} e^{-\left(\frac{\gamma}{a}\right)^{p}}}{\Gamma\left(\frac{d}{p}\right)}\right),
\end{aligned}
$$

where $\varphi($.$) is the lower incomplete Gamma function. It is$ worthy mentioning that, the CDF expression in (7) can be used also to calculate the outage probability of CI precoding technique. The outage probability is the probability that the received SNR, $\gamma_{k}$, falls below an acceptable threshold value, $\gamma_{t h}$. Therefore, we can obtain the outage probability of CI 
precoding by replacing $\gamma$ with $\gamma_{t h}$. Now the MGF of the received SNR, $\gamma_{k}$, can be derived as

$$
\mathcal{M}_{\gamma_{k}}(z)=\int_{0}^{\infty} e^{-z \gamma} f_{\gamma_{k}}(\gamma) d \gamma
$$

Substituting the PDF in (7) into (8), we can find

$$
\mathcal{M}_{\gamma_{k}}(z)=\int_{0}^{\infty} e^{-z \gamma}\left(\frac{\left(\frac{p}{a^{d}}\right) \gamma^{d-1} e^{-\left(\frac{\gamma}{a}\right)^{p}}}{\Gamma\left(\frac{d}{p}\right)}\right) d \gamma
$$

This MGF expression can be simplified using Gaussian Quadrature rules to

$\mathcal{M}_{\gamma_{k}}(z)=\sum_{i=1}^{n} \mathrm{H}_{i}\left(\frac{\left(\frac{p}{a^{d}}\right) e^{-(z-1) \gamma_{i}}\left(\gamma_{i}\right)^{d-1} e^{-\left(\frac{\gamma_{i}}{a}\right)^{p}}}{\Gamma\left(\frac{d}{p}\right)}\right)+R_{i}$,

where $\gamma_{i}$ and $\mathrm{H}_{i}$ are the $i^{\text {th }}$ zero and the weighting factor of the Laguerre polynomials, respectively, and the remainder $R_{i}$ is negligible for $n>15$ [34]. Alternatively, using Gamma distribution we can find

$$
\mathcal{M}_{\gamma_{k}}(z)=\int_{0}^{\infty} e^{-z \alpha_{k}|g|^{2}}\left(\frac{g^{N-1} e^{-g}}{(N-1) !}\right) d g,
$$

Using Gaussian Quadrature rules, the MGF can be obtained as

$$
\mathcal{M}_{\gamma_{k}}(z)=\sum_{i=1}^{n} \mathrm{H}_{i} e^{-z P_{p} \zeta_{k}\left|g_{i}\right|^{2}}\left(\frac{g_{i}^{N-1}}{(N-1) !}\right)+R_{i},
$$

where $\zeta_{k}=\frac{\left|\frac{\beta_{p}}{K} \mathbf{a}_{k} \mathbf{D u}\right|^{2}}{\sigma_{k}^{2}}, g_{i}$ here is the $i^{t h}$ zero of the Laguerre polynomials [34].

The average SNR of CI precoder can be obtained from the first derivative of $\mathcal{M}_{\gamma}(z)$ expressions evaluated at $z=0$. Hence, the average SNR can be calculated by

$$
\begin{gathered}
\bar{\gamma}_{k}=\left.\frac{\partial \mathcal{M}_{\gamma}(z)}{\partial z}\right|_{z=0}, \\
\bar{\gamma}_{k}=\sum_{i=1}^{n} \mathrm{H}_{i} \frac{\partial}{\partial z}\left(\frac{\left(\frac{p}{a^{d}}\right) e^{-(z-1) \gamma_{i}}\left(\gamma_{i}\right)^{d-1} e^{-\left(\frac{\gamma_{i}}{a}\right)^{p}}}{\Gamma\left(\frac{d}{p}\right)}\right)+R_{i} \mid
\end{gathered}
$$

Using a standard approach, the average of the SNR can be expressed as

$$
\bar{\gamma}_{k}=\int_{0}^{\infty} \gamma f_{\gamma_{k}}(\gamma) d \gamma
$$

Substituting the PDF in (7) into (15) we can get,

$$
\bar{\gamma}_{k}=\left(\frac{a \Gamma\left(\frac{1+d}{p}\right)}{\Gamma\left(\frac{d}{p}\right)}\right)=\frac{\alpha_{k}^{2} \Gamma(N+2)}{\Gamma(N)} .
$$

\section{Average Symbol Error Probability (SEP)}

In this section we calculate the average SEP for CI precoding with $M$-PSK signaling using a standard approach provided in literature [35], [36, (5.67)]. The average SEP of $M$-PSK can be calculated by [35, (5.67)]

$$
P_{e, k}=\frac{1}{\pi} \int_{0}^{\frac{\pi(M-1)}{M}} \mathcal{M}_{\gamma_{k}}\left(-\frac{\sin ^{2}\left(\frac{\pi}{M}\right)}{\sin ^{2} \Phi}\right) d \Phi .
$$

Next we will provide exact and approximated formulas to calculate the average SEP for MU-MISO transmission using CI precoding technique.

\section{A. Exact SEP}

The exact average SEP can be evaluated using the following Theorem.

Theorem 1. The exact analytical expression of the average SEP of the kth user can be calculated by

$$
\begin{gathered}
P_{e, k}=\frac{1}{\pi} \int_{0}^{\Theta}\left(\sum_{i=1}^{n} \frac{H_{i}}{z P_{p} \zeta_{k}}\left(\frac{\left(\frac{p}{a^{d}}\right)\left(\frac{v_{i}}{z P_{p} \zeta_{k}}\right)^{d-1} e^{-\left(\frac{v_{i}}{a z P_{p} \zeta_{k}}\right)^{p}}}{\Gamma\left(\frac{d}{p}\right)}\right)\right. \\
\left.+R_{i}\right) d \Phi
\end{gathered}
$$

and

$P_{e, k}=\frac{1}{\pi} \int_{0}^{\Theta}\left(\sum_{i=1}^{n} H_{i} e^{-z P_{p} \zeta_{k}\left|g_{i}\right|^{2}}\left(\frac{g_{i}^{N-1}}{(N-1) !}\right)+R_{i}\right) d \Phi$.

where $\Theta=\frac{\pi(M-1)}{M}$ and $z=-\frac{\sin ^{2}\left(\frac{\pi}{M}\right)}{\sin ^{2} \Phi}$ and the remainder $R_{i}$ is negligible for $n>15$.

Proof: The proof is provided in Appendix A.

As we can notice from the derived SEP equations, the derived SEP expressions are represented only with single integration which can be approximated efficiently using numerical integration methods.

\section{B. Approximate $S E P$}

In order to provide more insights, in the next Theorem we present very accurate closed-form approximation.

Theorem 2. The closed-form approximation of the average SEP of the kth legitimate user is presented in (20).

Proof: The proof is provided in Appendix B.

The numerical results show that the approximation expression in (20) is very tight to the exact one.

Remark 3. Firstly, the numerical results show that the approximation expression presented in Theorem 2 is very tight to the exact one presented in Theorem 1. In addition, from the analytical SEP expressions in Theorems 1, and 


$$
\begin{aligned}
P_{e, k}= & \left(\frac{\Theta}{2 \pi}-\frac{1}{6}\right) \sum_{i=1}^{n} \frac{\mathrm{H}_{i}}{\sin ^{2}\left(\frac{\pi}{M}\right) P_{p} \zeta_{k}}\left(\frac{\left(\frac{p}{a^{d}}\right)\left(\frac{\gamma_{i}}{\sin ^{2}\left(\frac{\pi}{M}\right) P_{p} \zeta_{k}}\right)^{d-1} e^{-\left(\frac{\gamma_{i}}{a z P_{p} \zeta_{k}}\right)^{p}}}{\Gamma\left(\frac{d}{p}\right)}\right) \\
& +\frac{1}{4} \sum_{i=1}^{n} \frac{3 \mathrm{H}_{i}}{4 \sin ^{2}\left(\frac{\pi}{M}\right) P_{p} \zeta_{k}}\left(\frac{\left(\frac{p}{a^{d}}\right)\left(\frac{3 \gamma_{i}}{4 \sin ^{2}\left(\frac{\pi}{M}\right) P_{p} \zeta_{k}}\right)^{d-1} e^{-\left(\frac{\gamma_{i}}{a z P_{p} \zeta_{k}}\right)^{p}}}{\Gamma\left(\frac{d}{p}\right)}\right) \\
+ & \left(\frac{\Theta}{2 \pi}-\frac{1}{4}\right) \sum_{i=1}^{n} \frac{\mathrm{H}_{i} \sin ^{2} \Theta}{\sin ^{2}\left(\frac{\pi}{M}\right) P_{p} \zeta_{k}}\left(\frac{\left(\frac{p}{a^{d}}\right)\left(\frac{\gamma_{i} \sin ^{2} \Theta}{\sin ^{2}\left(\frac{\pi}{M}\right) P_{p} \zeta_{k}}\right)^{d-1} e^{-\left(\frac{\gamma_{i}}{a z P_{p} \zeta_{k}}\right)^{p}}}{\Gamma\left(\frac{d}{p}\right)}\right)
\end{aligned}
$$

2 we can observe that as number of users or strength of the interference signals increases, the SEP at the users decreases unconventionally. This is because the interference signals have been designed to be constructive to the users. Furthermore, to further improve the SEP at the users, the transmit SNR, and number of BS antennas can be increased. Increasing the modulation order $M$ decreases the SEP at the users. The development of expressions in Theorems 1 and 2 will pave the way for optimizing practical approaches for interference exploitation to achieve the optimal performance. Please note that, all the derived expressions in this work are conditioned on the users locations, i.e., each user is located at a fixed distance from the BS. In case the users' locations are randomly distributed, the average SEP with respect to each user location can be calculated easily by averaging the derived SEP expressions over all possible user locations.

\section{Error Minimization Through Power ALLOCATION}

Equal power allocation (EPA) is not an optimal scheme for allocating the total transmission power between the users in communication systems, particularly when there is a notable disparity of channel strengths among the users. Therefore, the main aim of this section is to employ the above analytical results to improve the performance of the CI precoding technique with non-equal power allocation, under the assumption of total power constraint ${ }^{5}$. The considered approaches here seeking to explain the potential gain attained in the average SEP performance if the total available power is allocated more efficiently compared to the baseline EPA scheme. Firstly, we study power allocation scheme based on minimizing the sum symbol error probabilities, Min-Sum. In the second scheme we consider the power allocation based on minimizing the maximum SEP, Min-Max.

\footnotetext{
${ }^{5}$ Power allocation schemes that minimize the outage probability will be investigated in future work, in order to consider it in more details.
}

\section{A. Min-SUm SEP}

As we can see from the previous sections the derived SEP expressions are functions of the power allocated at the BS and thus this amount of power can be allocated in order to enhance the quality of the BS transmission. Here we consider the power allocation strategy that minimizes the total SEP of the considered system subject to the sumpower constraint. Accordingly, the corresponding optimization problem can be formulated as

$$
\begin{gathered}
\min _{\boldsymbol{a}} \mathbf{1}_{K}^{T} \mathfrak{p} \\
\text { S.t }: \sum_{k=1}^{K} a_{k}=1, a_{k} \geq 0,
\end{gathered}
$$

where $\mathfrak{p}=\left[P_{e, 1}, \ldots, P_{e, k}, \ldots, P_{e, K}\right]^{T}$ is the users SEP vector and $\boldsymbol{a}=\left[a_{1}, \ldots, a_{k}, \ldots, a_{K}\right]$ is the relative power allocation vector. By solving (21), the power allocation factor can be obtained as in Theorem 3 .

Theorem 4. The power allocation factor of user $k$ can be calculated by

$$
a_{k}=\frac{\zeta_{1} a_{1}}{\zeta_{k}}=\frac{1}{\zeta_{k} \sum_{k=1}^{K} \frac{1}{\zeta_{k}}} .
$$

Proof: The proof is provided in Appendix C.

In case the users have same path-loss, $\zeta_{1}=. .=\zeta_{k}=$ ..$\zeta_{K}$, (22) becomes $a_{k}=\frac{1}{K}$. This means that, under uniform path loss across the users the Min-Sum power allocation reduces to EPA.

\section{B. Min-Max SEP}

Min-Max power allocation scheme is a widely adopted as fairness criterion; thus, the obtained design by Min-Max scheme can provide high performance/fairness of the weak users. In the following, we study power allocation strategy 
to minimize the maximum SEP of the considered system subject to the sum-power constraint. Accordingly, the MinMax problem can be formulated as

$$
\begin{gathered}
\min _{\boldsymbol{a}} \quad \max \left\{P_{e, 1}, \ldots, P_{e, k}, \ldots P_{e, K}\right\} \\
\text { S.t }: \sum_{k=1}^{K} a_{k}=1, a_{k} \geq 0 .
\end{gathered}
$$

The optimization problem in (23) can be simplified as

$$
\begin{gathered}
\min _{a}\left(\frac{\Theta}{\pi}-\frac{1}{6}\right) \\
-c_{1}\left(\mathrm{H}_{1}\left(\prod_{k=1}^{K}\left[1-\frac{\varphi\left(d / p,\left(\bar{\gamma}_{1} / z_{1} a_{k} P_{p} \zeta_{k} \varrho_{k}\right)^{p}\right)}{\Gamma(d / p)}\right]\right)\right) \\
-c_{2}\left(\mathrm{H}_{1}\left(\prod_{k=1}^{K}\left[1-\frac{\varphi\left(d / p,\left(\bar{\gamma}_{1} / z_{2} a_{k} P_{p} \zeta_{k} \varrho_{k}\right)^{p}\right)}{\Gamma(d / p)}\right]\right)\right) \\
-c_{3}\left(\mathrm{H}_{1}\left(\prod_{k=1}^{K}\left[1-\frac{\varphi\left(d / p,\left(\bar{\gamma}_{1} / z_{3} a_{k} P_{p} \zeta_{k} \varrho_{k}\right)^{p}\right)}{\Gamma(d / p)}\right]\right)\right) \\
S . t: \sum_{k=1}^{K} a_{k}=1, a_{k} \geq 0
\end{gathered}
$$

where $c_{1}=\left(\frac{\Theta}{2 \pi}-\frac{1}{6}\right), c_{2}=\frac{1}{4}, c_{3}=\left(\frac{\Theta}{2 \pi}-\frac{1}{4}\right), z_{1}=$ $\sin ^{2}\left(\frac{\pi}{M}\right), z_{2}=\frac{4 \sin ^{2}\left(\frac{\pi}{M}\right)}{3}$ and $z_{3}=\frac{\sin ^{2}\left(\frac{\pi}{M}\right)}{\sin ^{2} \frac{\pi(M-1)}{M}}$.

Proof: The proof is provided in Appendix C.

The lower incomplete gamma function in (24) is given by

$$
\varphi(s, x)=\int_{0}^{x} r^{s-1} e^{-r} d r .
$$

It is noted that, the second derivation of the lower incomplete gamma function can be found as, $\frac{\partial^{2}}{\partial x} \varphi=$ $(s-x-1) e^{-x} x^{s-2}$. Since the convexity requires that the second derivative is not negative, this condition is satisfied of the lower incomplete gamma function only if $s>x-1$, which means that $\frac{d}{p}>\left(\frac{\bar{\gamma}_{1}}{z a_{k} P_{p} \zeta_{k} \varrho_{k}}\right)^{p}-1$, and $2 N>\sqrt{\left(\frac{\bar{\gamma}_{1}}{z a_{k} P_{p} \zeta_{k} \varrho_{k}}\right)}-1$. As we can see, this optimization problem in (24) is hard to solve numerically, and any closed form solution is hard if not impossible to find. However, some numerical software tools can be used to solve this problem such as Mathematica.

Remark 5. From the two power allocation schemes, it is noted that in low transmit SNR values Min-Sum scheme allocates high power to the best/ closest user to the BS and small amount of power to the far users. On the other hand, Min-Max scheme allocates relatively high power to the far user and small amount of power to the near users. In addition, as the transmit SNR value increases Min-Sum scheme starts gradually increasing the power allocated to the far users at the expense of the power allocated to the near users.

\section{Throughrut And Power EFFICIENCY}

In this section we consider the throughput and power efficiency of the CI precoding in MU-MISO systems. As the CI has been proposed to enhance the received SNR, it is important to consider and investigate the throughput performance of the CI technique. The throughput $(\tau)$ can be calculated using the following definition [37], [38]

$$
\tau=\left(1-P_{B}\right) \times c \times F \times K,
$$

where $P_{B}$ is the block error rate, $c=\log _{2}(M)$ is the bit per symbol and $F$ is the block length. The transmission in communication systems is generally based on sending blocks of $\mathscr{N}=c \times F$ sequential bits, where each block of $\mathscr{N}$ bits might represent sub or complete a user message. Therefore, the performance of such systems depends essentially on the probability of errors in each block. For coherent PSK modulation and in white Gaussian noise environment, the errors in each block are Binomially distributed. Thus, the probability of $q$ errors in one block can be expressed as [39]-[43]

$$
\operatorname{Pr}(q, \mathscr{N})=\left(\begin{array}{c}
\mathscr{N} \\
q
\end{array}\right) P_{b}^{q}\left(1-P_{b}\right)^{\mathscr{N}-q},
$$

where $P_{b}$ is the bit error probability (BEP) and can be calculated using the SEP derivation in Section IV. Consequently, the $P_{B}$ in fading channels for a block of $\mathscr{N}$ bits capable of correcting $Q$ errors can be written as [39]-[43]

$$
P_{B}=1-\sum_{q=0}^{Q}\left(\begin{array}{c}
\mathscr{N} \\
q
\end{array}\right) P_{b}^{q}\left(1-P_{b}\right)^{\mathscr{N}-q} .
$$

In case the receiver employs only error detection technique, a block is received correctly only if all $\mathscr{N}$ bits in the block are received successfully. Therefore, the overall system performance of such systems relies on the probability of occurrence of one or more bit errors in a block, i.e., $\operatorname{Pr}(0, \mathscr{N})$. On the other hand, if the receiver employs error-correction techniques which are able to correct up to $Q$ errors in a block, the system performance is dominated by the probability of occurrence of more than $Q$ errors in a block, i.e., $\operatorname{Pr}(Q, \mathscr{N})$. In case when $Q=0$ and $\mathscr{N}=1$, $P_{B}$ becomes the BEP [39], [40], [42], [43] . This definition of the $P_{B}$ has been widely studied in literature, for instance [39], [40], [42], [43] . For simplicity and mathematical tractability we employ the below approximate expression to derive the BEP from our SEP derivation above [35], [44, (8.119)]

$$
P_{b} \simeq \frac{2}{\max \left(\log _{2} M, 2\right)} \sum_{i=1}^{\max \left(\frac{M}{4}, 1\right)} \frac{1}{\pi}
$$




$$
\times \int_{0}^{\pi / 2} \mathcal{M}_{\gamma}\left(-\frac{\log _{2} M}{\sin ^{2} \theta} \sin ^{2} \frac{(2 i-1) \pi}{M}\right) d \theta,
$$

where $\simeq$ denotes approximation expression. Substituting (10) into (29) we can get

$$
\begin{aligned}
& P_{b} \simeq \frac{2}{\max \left(\log _{2} M, 2\right)} \sum_{i=1}^{\max \left(\frac{M}{4}, 1\right)} \frac{1}{\pi} \\
& \times \sum_{i=1}^{n} \frac{\mathrm{H}_{i}\left(\frac{p}{a^{d}}\right)\left(\gamma_{i}\right)^{d-1} e^{-\left(\frac{\gamma_{i}}{a}\right)^{p}}}{\Gamma\left(\frac{d}{p}\right)} \\
& \int_{0}^{\pi / 2} e^{-\left(-\frac{\log _{2} M}{\sin ^{2} \theta} \sin ^{2} \frac{(2 i-1) \pi}{M}-1\right) \gamma_{i}} d \theta .
\end{aligned}
$$

which can be found as

$$
\begin{gathered}
P_{b} \simeq \frac{2}{\max \left(\log _{2} M, 2\right)} \sum_{i=1}^{\max \left(\frac{M}{4}, 1\right)} \frac{1}{\pi} \\
\times \sum_{i=1}^{n} \mathrm{H}_{i}\left(\frac{\left(\frac{p}{a^{d}}\right)\left(\gamma_{i}\right)^{d-1} e^{-\left(\frac{\gamma_{i}}{a}\right)^{p}}}{\Gamma\left(\frac{d}{p}\right)}\right) \\
\left(\frac{\pi}{2} e^{\gamma_{i}} \operatorname{Erfc}\left(\sqrt{-\log _{2} M \times \sin ^{2} \frac{(2 i-1) \pi}{M} \gamma_{i}}\right)\right) .
\end{gathered}
$$

Finally, substituting the BEP expression in (31) into (28) and then into (26) we can find the system throughput. Similarly, in the communication systems where the decoding depends on the symbol error, the $P_{B}$ can be evaluated using the SEP. In this case we can define $\mathscr{N}$ as number of symbols in each block and $Q$ as number of symbol errors, thus $P_{B}$ can be evaluated by replacing $P_{b}$ with $P_{e}$ in (28) [41], [45]. In the special case when $Q=0$ and $\mathscr{N}=1$, $P_{B}$ becomes the SEP. Hence, the throughput in this case can be calculated as in the following expression [41], [45]

$$
\begin{gathered}
\tau=\left(\sum_{q=0}^{Q}\left(\begin{array}{c}
\mathscr{N} \\
q
\end{array}\right) P_{e, k}^{q}\left(1-P_{e . k}\right)^{\mathscr{N}-q}\right) \\
\times \log _{2}(M) \times \mathscr{N} \times K,
\end{gathered}
$$

where the exact $P_{e . k}$ is given in (18), and the approximate $P_{e . k}$ is given (20).

The derived expression of the throughput can be used now to calculate the power efficiency (PE). The power efficiency combines both the throughput with the power consumption at the BS, and can be expressed as [38]

$$
\mathrm{PE}=\frac{\tau}{P_{t o t}},
$$

where $P_{t o t}$ is the total power consumed during the transmission. In practical systems, the total power can be calculated by [46]-[48]

$$
P_{t o t}=\frac{P_{P A}+P_{R F}+P_{D S}}{\left(1-\varsigma_{D C}\right)\left(1-\varsigma_{M S}\right)\left(1-\varsigma_{\text {cool }}\right)},
$$

where $\varsigma_{D C}, \varsigma_{M S}$ and $\varsigma_{c o o l}$ represent the losses of the DCDC supply, main power supply and the active cooling, respectively [46], [47]. In addition, $P_{P A}$ is the average power consumption of the amplifiers and given by $P_{P A}=$ $\frac{P_{P}}{\eta_{p a}}$, where $\eta_{p a}$ is the efficiency of the power amplifiers. Furthermore, $P_{R F}$ is the power consumption of the other electronic components in the RF chains, and can be written as $P_{R F}=N\left(P_{D}+P_{m}+P_{f}\right)+P_{s y}$, where $P_{D}, P_{m}$ and $P_{f}$ are the power consumption of the digital-to-analog converters, signal mixers and filters, respectively, while $P_{s y}$ is the power consumption at the frequency synthesizer. Moreover, $P_{D S}$ is the power consumed by the digital signal processor [46]-[48]. At high SNR values, the error rate will go to zero $P_{B} \rightarrow 0$, and the throughput expression converges to

$$
\tau=c \times F \times K .
$$

Accordingly, the power efficiency converges to

$$
\mathrm{PE}=\frac{c \times F \times K}{P_{\text {tot }}} .
$$

Therefore, in this case the adaptive modulation would be required to switch to a higher order modulation for higher SNR values.

\section{NUMERICAL RESULTS}

This section presents simulation and numerical results of the derived expressions in this paper. Monte-Carlo simulations are performed with $10^{6}$ independent trials. It is assumed that, the users have same noise power, $\sigma^{2}$, and thus the transmit SNR $\left(\eta_{t}\right)$ is defined as $\eta_{t}=\frac{P_{p}}{\sigma^{2}}$. In addition, the path-loss exponent in this section is chosen to be $m=2.7$.

Firstly, in Fig. 2 we plot the CDF of the received SNR at the $k^{t h}$ user for different values of the transmit SNR, $\eta_{t}$, number of users, $K$, number of BS antennas, $N$, and the vector $\mathbf{u}$. The analytical and simulation results are in well agreement, which confirms the accuracy of the distribution considered in Section (III). In addition, from these results it is clear that, the values of the elements of $\mathbf{u}$ have impact on the CDF and thus on the system performance in general. In this regard it is noted that, user $k$ can achieve the optimal performance when $u_{k}=1$, which is the case presented in Figs. 2a and 2b. Furthermore, the CDF of the received SNR for different values of $N$ and $K$ when the elements of $\mathbf{u}$ have same value, $u_{k}=\frac{1}{K}$, are presented in Figs. 2 c, 2 d, and $2 \mathrm{e}$ and when $u_{k}$ has the smallest value is presented in Fig. 2f. In all these cases the variance of the received SNR will be reduced by the value of $u_{k}$, and thus smaller value 


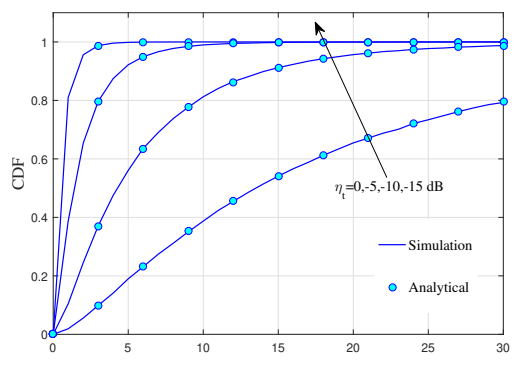

(a) The CDF of the received SNR for different transmit SNR values, $\eta_{t}$, when $N=$ $K=4$, and $\mathbf{u}=\mathbf{a}_{k}$.

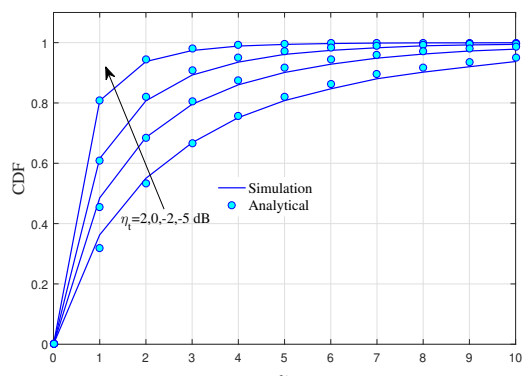

(d) The CDF of the received SNR for different transmit SNR values, $\eta_{t}$, when $N=$ $K=3$, and $\mathbf{u}=\frac{1}{K} \mathbf{1}$.

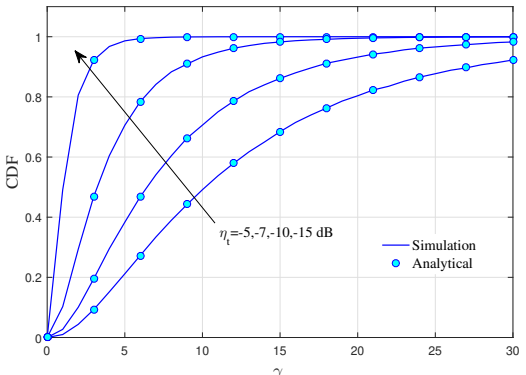

(b) The CDF of the received SNR for different transmit SNR values, $\eta_{t}$, when $N=$ $6, K=4$, and $\mathbf{u}=\mathbf{a}_{k}$.

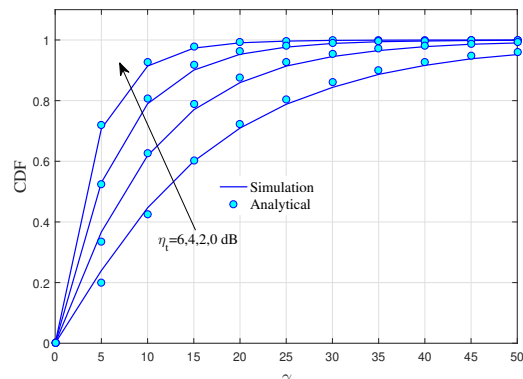

(e) The CDF of the received SNR for different transmit SNR values, $\eta_{t}$, when $N=$ $8, K=5$, and $\mathbf{u}=\frac{1}{K} \mathbf{1}$.

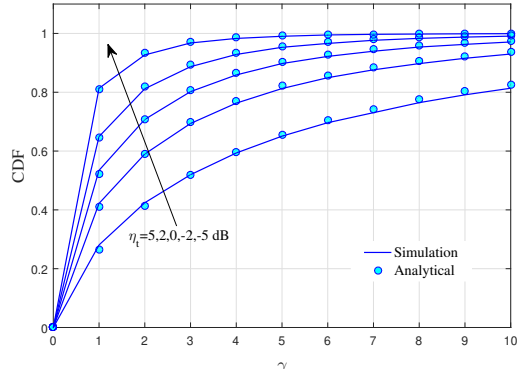

(c) The CDF of the received SNR for different transmit SNR values, $\eta_{t}$, when $N=$ $K=2$, and $\mathbf{u}=\frac{1}{K} \mathbf{1}$.

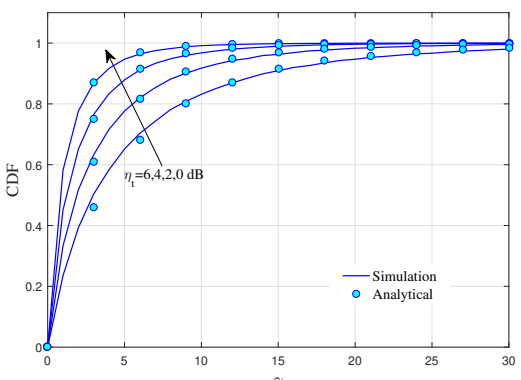

(f) The CDF of the received SNR for different transmit SNR values, $\eta_{t}$, when $N=K=3$, and $u_{k}=0.2$.

Figure 2: The CDF of the received SNR for different values of the transmit SNR, $\eta_{t}$, number of users $K$, number of BS antennas $N$ and $\mathbf{u}$.

of $u_{k}$ will result in poorer/weaker performance/SNR of user $k$ in the system. Finally, it is worthy mentioning that, the results presented in Fig. 2, can be used also to present the outage probability of CI precoding technique.

Fig. 3, illustrates the average received SNR versus the transmit SNR, $\eta_{t}$, for different values of $N$ and $K$. Fig. $3 \mathrm{a}$, presents the average received SNR when $K=4$ and Fig. $3 b$, shows the average received SNR when $K=2$. The good matching between the results confirms the derived expressions in Section (III). Generally and as anticipated, increasing the transmit SNR and number of antennas lead to enhance the average received SNR. In addition, interference exploitation technique is most useful in high interference scenarios, where there is significant interference to exploit. Therefore, increasing number of users results in increasing number of the constructive interferences and thus enhancing the received SNR. Furthermore, the gain attained by increasing number of the antennas is almost fixed with the transmit SNR in the all considered scenarios.

Fig. 4, shows the exact and approximated average SEP versus transmit SNR, $\eta_{t}$, for BPSK, QPSK and 8-PSK, when the distances are normalized to one, i.e, without the impact of the path-loss $d_{1}=d_{2}=d_{3}=d_{4}=1 \mathrm{~m}$. Fig. 4a, presents the average SEP when $N=K=4$, and Fig. 4b, illustrates the average SEP when $N=6$, and $K=4$. Additionally and for sake of comparison, some results of minimum-mean-squared-error (MMSE) and ZF precoders with symbol-level power normalization, are also included in these figures. It should be pointed out that the analytical results in these figures are obtained from the expressions derived in Section (IV). Several interesting points can be extracted from this figure. Firstly, it is evident that the SEP reduces with increasing the transmit SNR, $\eta_{t}$, and CI precoding technique always outperforms the MMSE technique in the all SNR values with an up to $15 \mathrm{~dB}$ gain in the transmit SNR for a given SEP. For instance, in case $N=K=4$, and 8-PSK, the CI achieves SEP $=10^{-2}$ with $\eta_{t}=15 \mathrm{~dB}$ while MMSE performs $\mathrm{SEP}=10^{-2}$ with $\eta_{t}=30 \mathrm{~dB}$. In addition, it is clear that the approximated results obtained from Section (IV-B) are very tight to the exact ones. Finally, comparing Fig. 4a and Fig. 4b, we can see that, increasing number of BS antennas always enhances the average SEP, and reduces the gap performance between the two precoding techniques.

In order to investigate the impact of number of users and number of BS antennas on the average SEP, in Fig. 5 we present the average SEP for the CI, ZF and MMSE precoding techniques for BPSK, QPSK and 8PSK, when $N=K=6$, as in Fig. 5a and when $N=8, K=6$ as in Fig. 5b, when the distances are normalized to one, .i.e, without the impact of the path-loss $d_{1}=d_{2}=$ $d_{3}=d_{4}=1 \mathrm{~m}$. From the results in Figs. 5 and 4 , it is obvious that increasing number of BS antennas $N$ and/or number of users $K$ result in enhancing the system 


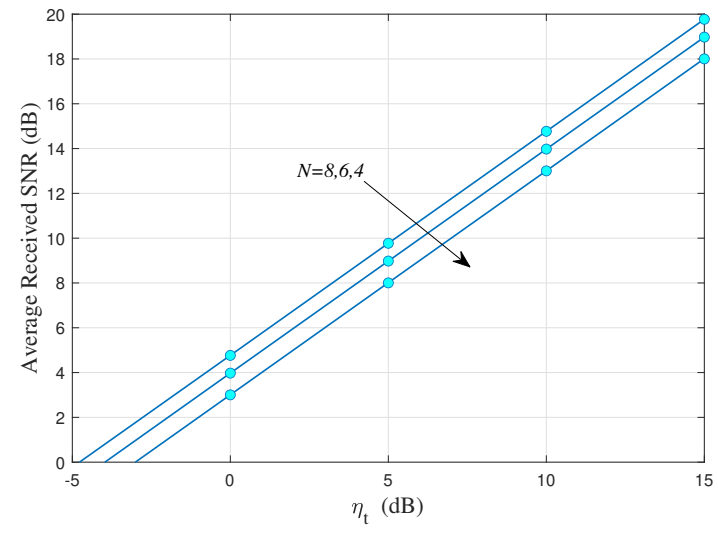

(a) Average received SNR versus transmit $\mathrm{SNR}, \eta_{t}$, for different values of $N$ when $K=4$.

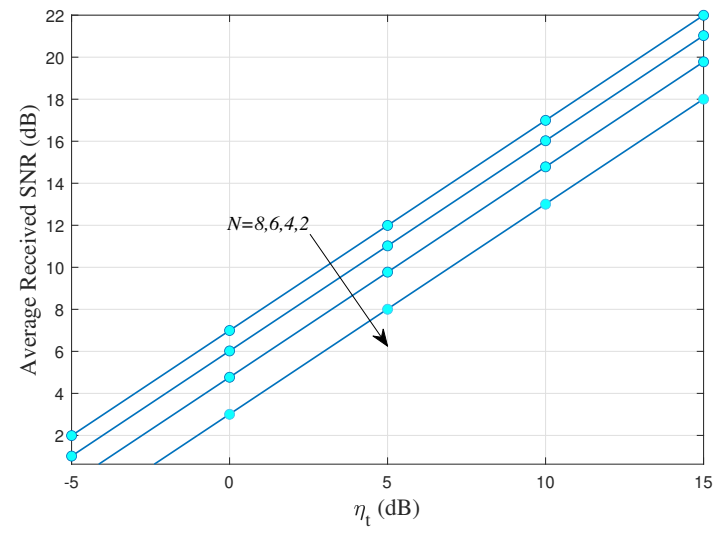

(b) Average received SNR versus transmit $\mathrm{SNR}, \eta_{t}$, for different values of $N$ when $K=2$.

Figure 3: Average received SNR versus transmit SNR, $\eta_{t}$, for different values of $N$ and $K$.

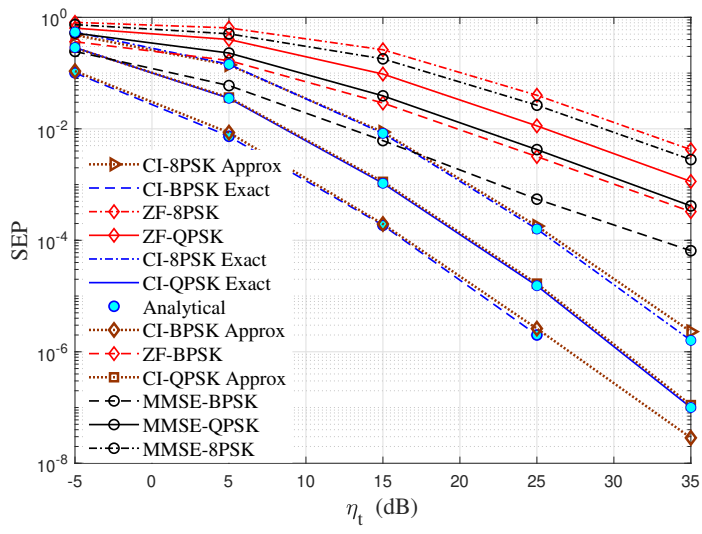

(a) SEP versus transmit SNR, $\eta_{t}$, with different types of input, when $N=K=4$.

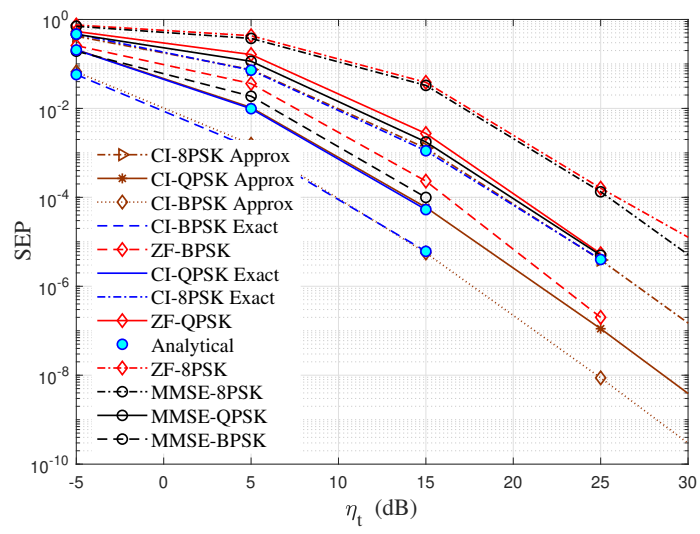

(b) SEP versus transmit SNR, $\eta_{t}$, with different types of input, when $N=6, K=4$.

Figure 4: SEP versus transmit SNR for various input types, when $N=4,6$ and $K=4$.

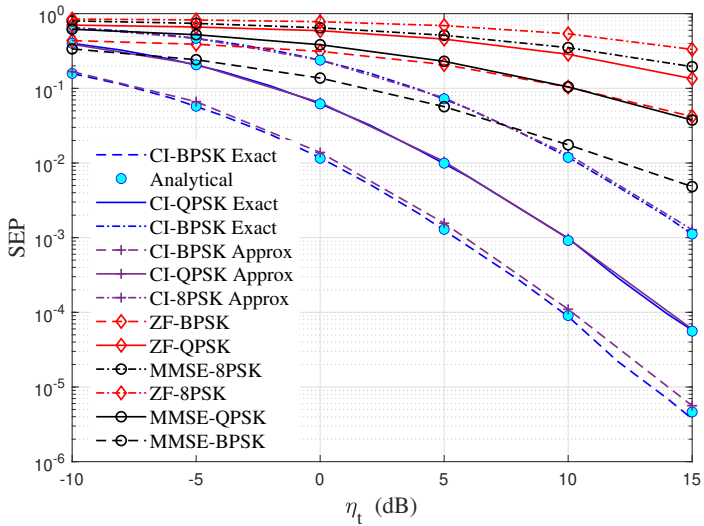

(a) SEP versus transmit SNR, $\eta_{t}$, with different types of input, when $N=K=6$.

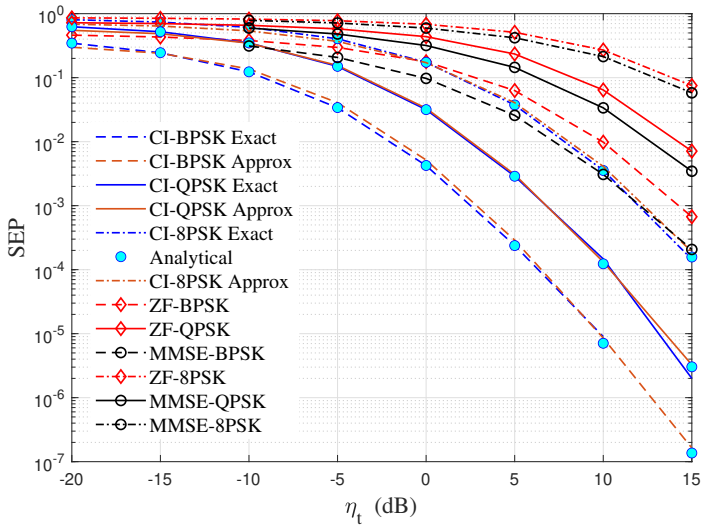

(b) SEP versus transmit SNR, $\eta_{t}$, with different types of input, when $N=8, K=6$.

Figure 5: SEP versus transmit SNR for various input types, when $N=6,8$ and $K=6$. 


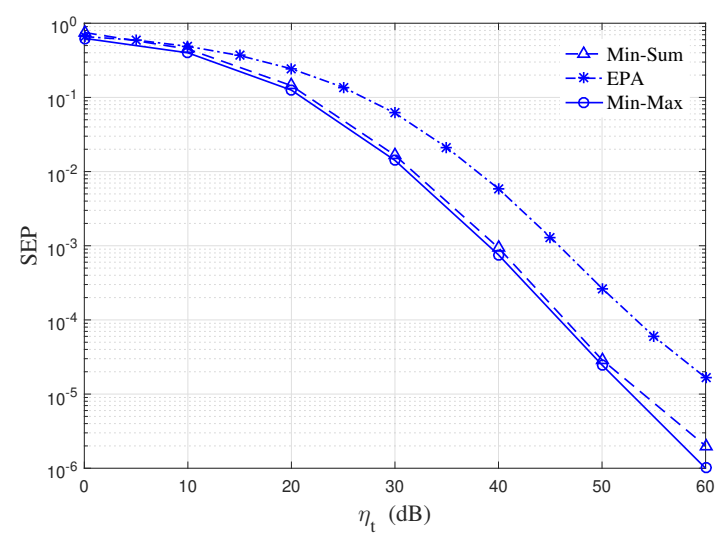

(a) SEP versus transmit SNR, $\eta_{t}$, with different power allocation schemes and QPSK input, when $N=K=3$.

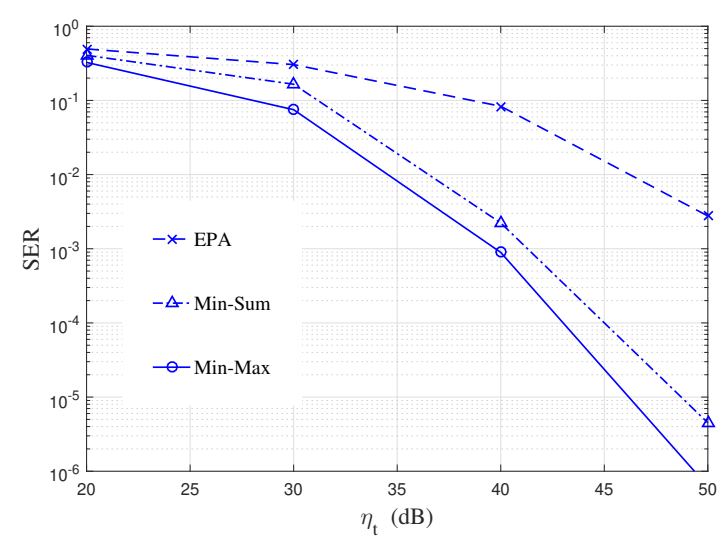

(b) SEP versus transmit SNR, $\eta_{t}$, with different power allocation schemes and QPSK input, when $N=K=8$.

Figure 6: SEP versus transmit SNR with different power allocation schemes.

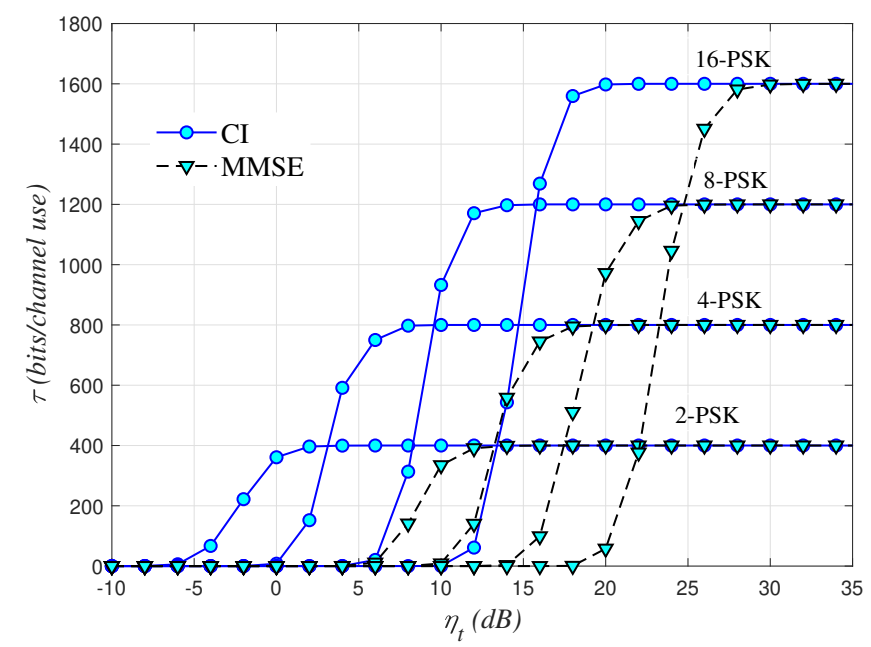

Figure 7: Throughput versus transmit SNR, $\eta_{t}$, for various input types, when $\mathscr{N}=100, Q=5$, and $N=K=4$.

performance. Furthermore, the CI precoding has always better performance than MMSE in the all SNR values with an up to $20 \mathrm{~dB}$ gain in the transmit SNR for a given SEP. In addition, comparing the average SEP in Fig. 5a and Fig. 5 b, similar observations can be concluded as in the previous case when $K=4$.

Fig. 6 illustrates the average SEP versus the transmit SNR, $\eta_{t}$, for different power allocation schemes, EPA, MinSum and Min-Max schemes. Fig. 6a, presents the average SEP versus $\eta_{t}$ when $N=K=3$, while Fig. 6 b, presents the average SEP versus $\eta_{t}$ when $N=K=8$, and the users are randomly distributed. From this figure it can be observed that, EPA scheme always results in the highest SEP in the all cases. Therefore, we can say EPA scheme provides the lower bound of the average SEP for the considered MUMISO system. In addition, looking closer at the results in Fig. $6 \mathrm{a}$ and Fig. $6 \mathrm{~b}$ one can clearly observe that, the SEP is dominated by the performance of the worst user, and thus the Min-Max scheme has the best performance.

In Fig. 7 we present the throughput versus the transmit
SNR, $\eta_{t}$, for different types of input, BPSK, QPSK, 8PSK and 16-PSK. For sake of comparison, results of the conventional MMSE precoding technique are included in the figure. The results in this figure are obtained from the expressions provided in Section (VI). The throughput saturates to the value of, $\log _{2}(M) \times \mathscr{N} \times K$, past a certain transmit SNR $\eta_{t}$ value, the throughput saturates at 400 bits/channel use in BPSK, at 800 bits/channel use in QPSK, at $1200 \mathrm{bits} / \mathrm{channel}$ use in 8-PSK and at $1600 \mathrm{bits} / \mathrm{channel}$ use in 16-PSK. Furthermore, the CI precoding outperforms the conventional MMSE scheme for a wide range with an up to $10 \mathrm{~dB}$ gain in the transmit SNR for a given throughput value. Finally and as anticipated, in low SNR values the lower modulation orders have better performance than the higher ones, for instance at $0 \mathrm{~dB}$ BPSK achieves the highest throughput. However, in high SNR values the higher modulation orders achieve better performance, for instance at $20 \mathrm{~dB}$ 16-PSK has optimal performance.

Finally, Fig. 8 depicts the power efficiency as function of number of BS antennas, $N$, for different values of 


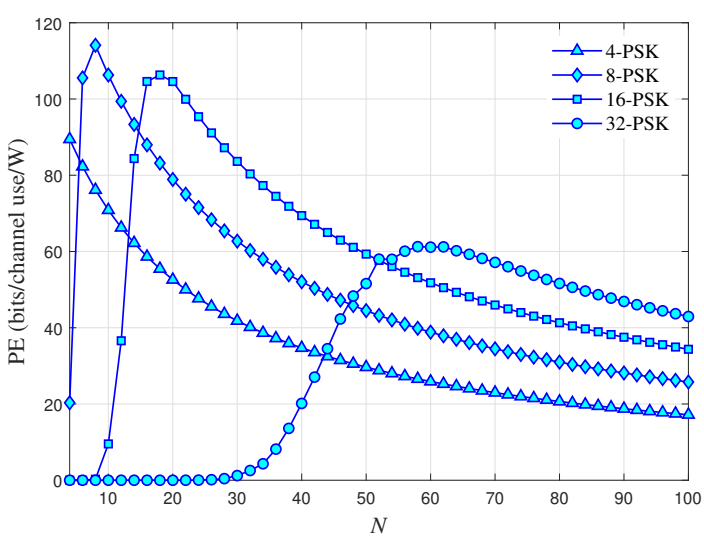

(a) Power Efficiency versus number of BS antennas, $N$, for various input types, when $\mathscr{N}=100, Q=10$, and $K=4$.

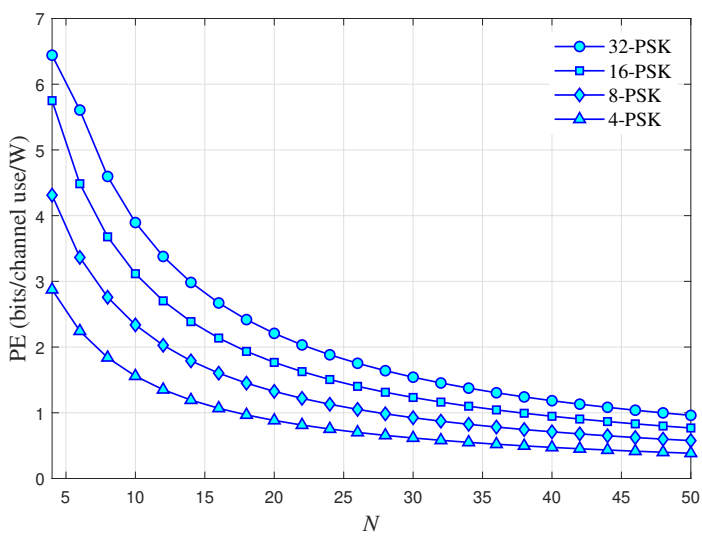

(b) Power Efficiency versus number of BS antennas, $N$, for various input types, when $\mathscr{N}=100, Q=10$, and $K=4$.

Figure 8: Power Efficiency versus number of BS antennas, $N$, for different values of the transmission power.

the transmission power. The results in these figures are obtained from the power efficiency expression provided in Section (VI). In Fig. 8a we present the power efficiency versus $N$ when $\varsigma_{D C}=0.075, \varsigma_{M S}=0.09, P_{P}=35 \mathrm{dbm}$, $\eta_{p a}=0.8, P_{D}=7.8 \mathrm{~mW}, P_{m}=15.2 \mathrm{~mW}, P_{f}=10 \mathrm{~mW}$, $P_{s y}=25 \mathrm{~mW}$, and $P_{D S}=2 \mathrm{~W}$ [46]-[48]. From Fig. 8a we can observe that when number of BS antennas is small the lower modulation orders achieve higher power efficiency than the higher orders, for instance when $N=4$ QPSK has best performance. On the other hand, when number of BS antennas is large the higher modulation orders become better than the lower ones, for instance 32-PSK achieves the highest power efficiency when $N=60$. Furthermore, in order to clearly demonstrate the impact of transmission power on the power efficiency for different types of input, we plot in Fig. 8b the power efficiency versus $N$ when the transmission power is very high $P_{P}=20 \mathrm{dbW}$. In this case, the higher modulation orders always have better system performance regardless number of antennas implemented at the BS. Furthermore, comparing Figs. 8a and $8 \mathrm{~b}$ it can be concluded that, the power efficiency achieved in low transmit SNR is much higher than that in high transmit SNR regime.

\section{CONCLUSIONS}

In this paper the statistics of the received SNR of CI precoding technique has been considered for the first time. Firstly, exact closed form expressions of the MGF and the average received SNR have been derived. Then, the derived MGF expression was used to calculate the average SEP. In light of this, exact average SEP expression for CI precoding with $M$-PSK was obtained. In addition, accurate asymptotic approximation for the average SEP has been provided. Building on the new performance analysis, different power allocation schemes to enhance the average SEP have been considered. In the first scheme, power allocation technique based on minimizing the total SEP was studied, while in the second scheme power allocation technique based on minimizing the maximum SEP was investigated. Furthermore, new and explicit analytical expressions of the throughput and power efficiency of the CI precoding in MUMISO systems have been derived. The results in this paper explained that the $\mathrm{CI}$ scheme outperforms $\mathrm{ZF}$ scheme in the all considered metrics. Furthermore, increasing the transmit SNR, number of users and number of BS antennas always enhance the achieved SEP. It was also shown that, using EPA leads to the highest SEP and the considered power allocation techniques can perform very low SEP. Finally, in low transmit SNR values and when number of BS antennas is small, the lower modulation orders achieve higher power efficiency than the higher modulation orders.

\section{APPENDIX A}

For simplicity (17) can be written as

$$
P_{e, k}=\frac{1}{\pi} \int_{0}^{\Theta} \mathcal{M}_{\gamma_{k}}(z) d \Phi
$$

where $\Theta=\frac{\pi(M-1)}{M}$ and $z=-\frac{\sin ^{2}\left(\frac{\pi}{M}\right)}{\sin ^{2} \Phi}$. By substituting (9) into (37), we can obtain the average SEP as

$$
P_{e, k}=\frac{1}{\pi} \int_{0}^{\Theta}\left(\int_{0}^{\infty} e^{-z \gamma}\left(\frac{\left(\frac{p}{a^{d}}\right) \gamma^{d-1} e^{-\left(\frac{\gamma}{a}\right)^{p}}}{\Gamma\left(\frac{d}{p}\right)}\right) d \gamma\right) d \Phi
$$

Applying Gaussian Quadrature rules we can find (18). Using Gamma distribution, substituting (11) into (37) we can also find the average SEP as

$$
P_{e, k}=\frac{1}{\pi} \int_{0}^{\Theta}\left(\int_{0}^{\infty} e^{-z \alpha_{k}|g|^{2}}\left(\frac{g^{N-1} e^{-g}}{(N-1) !}\right) d g\right) d \Phi
$$

Applying Gaussian Quadrature rules we can obtain (19). 


\section{APPENDIX B}

Here we derive an approximation expression of the average SEP of the considered scenario. Firstly, (37) can be written as

$$
\begin{aligned}
P_{e, k} & =\mathcal{E}\left[\frac{1}{\pi} \int_{0}^{\frac{\pi}{2}} \exp \left(-\frac{\sin ^{2}\left(\frac{\pi}{M}\right)}{\sin ^{2} \Phi}\right) d \Phi\right. \\
& \left.+\frac{1}{\pi} \int_{\frac{\pi}{2}}^{\Theta} \exp \left(-\frac{\sin ^{2}\left(\frac{\pi}{M}\right)}{\sin ^{2} \Phi}\right) d \Phi\right],
\end{aligned}
$$

where $\mathcal{E}[$.$] is the average operation. Now, the first term in$ (40) can be approximated by [36], [49]

$$
\begin{gathered}
\frac{1}{\pi} \int_{0}^{\frac{\pi}{2}} \exp \left(-\frac{\sin ^{2}\left(\frac{\pi}{M}\right)}{\sin ^{2} \Phi}\right) d \Phi \approx \\
\frac{1}{12} e^{\left(-\sin ^{2}\left(\frac{\pi}{M}\right)\right)}+\frac{1}{4} e^{\left(-\frac{4 \sin ^{2}\left(\frac{\pi}{M}\right)}{3}\right)} .
\end{gathered}
$$

Similarly, the second term in (40) can be approximated as [36], [49]

$$
\begin{gathered}
\frac{1}{\pi} \int_{\frac{\pi}{2}}^{\Theta} \exp \left(-\frac{\sin ^{2}\left(\frac{\pi}{M}\right)}{\sin ^{2} \Phi}\right) d \Phi \approx \\
\frac{1}{2 \pi}\left(e^{\left(-\sin ^{2}\left(\frac{\pi}{M}\right)\right)}+\frac{1}{4} e^{\left(-\frac{\sin ^{2}\left(\frac{\pi}{M}\right)}{\sin ^{2} \Theta}\right)}\right)\left(\Theta-\frac{\pi}{2}\right) .
\end{gathered}
$$

Now substituting (41) and (42) into (40), we can obtain approximated expression of SEP as [36], [49]

$$
\begin{gathered}
P_{e, k}=\mathcal{E}\left[\frac{1}{12} e^{\left(-\sin ^{2}\left(\frac{\pi}{M}\right)\right)}+\frac{1}{4} e^{\left(-\frac{4 \sin ^{2}\left(\frac{\pi}{M}\right)}{3}\right)}\right. \\
\left.+\frac{1}{2 \pi}\left(e^{\left(-\sin ^{2}\left(\frac{\pi}{M}\right)\right)}+\frac{1}{4} e^{\left(-\frac{\sin ^{2}\left(\frac{\pi}{M}\right)}{\sin ^{2} \Theta}\right)}\right)\left(\Theta-\frac{\pi}{2}\right)\right]
\end{gathered}
$$

which can be written as

$$
\begin{gathered}
P_{e, k}=\frac{1}{12} \mathcal{M}_{\gamma}\left(\sin ^{2}\left(\frac{\pi}{M}\right)\right)+\frac{1}{4} \mathcal{M}_{\gamma}\left(\frac{4 \sin ^{2}\left(\frac{\pi}{M}\right)}{3}\right) \\
+\frac{1}{2 \pi}\left(\mathcal{M}_{\gamma}\left(\sin ^{2}\left(\frac{\pi}{M}\right)\right)+\frac{1}{4} \mathcal{M}_{\gamma}\left(\frac{\sin ^{2}\left(\frac{\pi}{M}\right)}{\sin ^{2} \Theta}\right)\right)\left(\Theta-\frac{\pi}{2}\right) .
\end{gathered}
$$

$$
\begin{gathered}
P_{e, k}=\left(\frac{\Theta}{2 \pi}-\frac{1}{6}\right) \mathcal{M}_{\gamma}\left(\sin ^{2}\left(\frac{\pi}{M}\right)\right)+\frac{1}{4} \mathcal{M}_{\gamma}\left(\frac{4 \sin ^{2}\left(\frac{\pi}{M}\right)}{3}\right) \\
+\left(\frac{\Theta}{2 \pi}-\frac{1}{4}\right) \mathcal{M}_{\gamma}\left(\frac{\sin ^{2}\left(\frac{\pi}{M}\right)}{\sin ^{2} \Theta}\right)
\end{gathered}
$$

Finally using the derived formula in (45), the approximated expression of the average SEP for MU-MISO system using CI precoding technique can be written as in Theorem 2 .

\section{APPENDIX C}

This optimization problem in (21) can be formulated in a simpler way as

$$
\begin{gathered}
\min _{\boldsymbol{a}} \sum_{k=1}^{K} P_{e, k} \\
\text { S.t }: \sum_{k=1}^{K} a_{k}=1, a_{k} \geq 0 .
\end{gathered}
$$

For simplicity, substituting (12) into the derived SEP expression in (45) and (46), we can get

$$
\begin{gathered}
\min _{a_{k}} \sum_{k=1}^{K}\left\{c_{1}\left[\sum_{i=1}^{n} \vartheta_{i} e^{-z_{1} a_{k} P_{p} \zeta_{k}\left|g_{i}\right|^{2}}\right]+c_{2}\left[\sum_{i=1}^{n} \vartheta_{i} e^{-z_{2} a_{k} P_{p} \zeta_{k}\left|g_{i}\right|^{2}}\right]\right. \\
\left.+c_{3}\left[\sum_{i=1}^{n} \vartheta_{i} e^{-z_{3} a_{k} P_{p} \zeta_{k}\left|g_{i}\right|^{2}}\right]\right\} \\
\text { S.t }: \sum_{k=1}^{K} a_{k}=1, a_{k} \geq 0
\end{gathered}
$$

where $c_{1}=\frac{\left(\frac{(M-1)}{2 M}-\frac{1}{6}\right)}{(N-1) !}, c_{2}=\frac{1}{4(N-1) !}, c_{3}=\frac{\left(\frac{(M-1)}{2 M}-\frac{1}{4}\right)}{(N-1) !}$, $\vartheta_{i}=g_{i}^{N-1} \mathrm{H}_{i}, z_{1}=\sin ^{2}\left(\frac{\pi}{M}\right), z_{2}=\frac{4 \sin ^{2}\left(\frac{\pi}{M}\right)}{3}$ and $z_{3}=\frac{\sin ^{2}\left(\frac{\pi}{M}\right)}{\sin ^{2} \frac{\pi(M-1)}{M}}$. The function in (47) is convex in the parameters $a_{k}$ over the feasible set defined by linear power ratio constraints, $\frac{\partial^{2}}{\partial a_{k}^{2}} P_{e, k}>0$ for $a_{k}>0$. Therefore, the optimization problem (47) can be solved using CVX and other numerical software tools. In order to develop some insights for the power allocation policy we can consider numerical solution of this problem as follows. Following the definitions in [50], the Lagrangian of this optimization problem in (47) can be written as,

$$
\mathfrak{L}(\mathfrak{p}, \lambda)=\mathbf{1}_{K}^{T} \mathfrak{p}+\lambda\left(\sum_{k=1}^{K} a_{k}-1\right),
$$

where $\lambda$ is the Lagrange multiplier satisfying the power constraint. Therefore, the power allocation solution can be found from the conditions

and 


$$
\begin{gathered}
\frac{\partial}{\partial a_{k}} \mathfrak{L}(\mathfrak{p}, \lambda)=\lambda-\psi_{k}=0, \\
\frac{\partial}{\partial \lambda} \mathfrak{L}(\mathfrak{p}, \lambda)=\left(\sum_{k=1}^{K} a_{k}-1\right)=0,
\end{gathered}
$$

where $\psi_{k}=c_{1}\left[\sum_{i=1}^{n} \omega_{i 1, k} \vartheta_{i} e^{-\omega_{i 1, k} a_{k}}\right]+$ $c_{2}\left[\sum_{i=1}^{n} \omega_{i 2, k} \vartheta_{i} e^{-a_{k} \omega_{i 2, k}}\right]+c_{3}\left[\sum_{i=1}^{n} \omega_{i 3, k} \vartheta_{i} e^{-a_{k} \omega_{i 3, k}}\right]$, $\omega_{i j, k}=z_{j} P_{p} \zeta_{k}\left|g_{i}\right|^{2}, j=1,2,3$. From (49), we can notice that $\psi_{k}=\psi_{k-1}=\ldots=\psi_{1}$, so that

$$
\begin{array}{r}
c_{1}\left[\sum_{i=1}^{n} \omega_{i 1, k} \vartheta_{i} e^{-\omega_{i 1, k} a_{k}}\right]+c_{2}\left[\sum_{i=1}^{n} \omega_{i 2, k} \vartheta_{i} e^{-a_{k} \omega_{i 2, k}}\right] \\
+c_{3}\left[\sum_{i=1}^{n} \omega_{i 3, k} \vartheta_{i} e^{-a_{k} \omega_{i 3, k}}\right] \\
=c_{1}\left[\sum_{i=1}^{n} \omega_{i 1,1} \vartheta_{i} e^{-\omega_{i 1,1} a_{1}}\right]+c_{2}\left[\sum_{i=1}^{n} \omega_{i 2,1} \vartheta_{i} e^{-a_{1} \omega_{i 2,1}}\right] \\
+c_{3}\left[\sum_{i=1}^{n} \omega_{i 3,1} \vartheta_{i} e^{-a_{1} \omega_{i 3,1}}\right] .
\end{array}
$$

Considering the first-order Laguerre polynomial, we can get

$$
\begin{gathered}
c_{1}\left[\omega_{11, k} e^{-\omega_{11, k} a_{k}}\right]+c_{2}\left[\omega_{12, k} e^{-a_{k} \omega_{12, k}}\right] \\
+c_{3}\left[\omega_{13, k} e^{-a_{k} \omega_{13, k}}\right] \\
=c_{1}\left[\omega_{11,1} e^{-\omega_{11,1} a_{1}}\right]+c_{2}\left[\omega_{12,1} e^{-a_{1} \omega_{12,1}}\right] \\
+c_{3}\left[\omega_{13,1} e^{-a_{1} \omega_{13,1}}\right] .
\end{gathered}
$$

and

$$
\begin{gathered}
\left(\frac{(M-1)}{2 M}-\frac{1}{6}\right) \zeta_{k} e^{-z_{1} P_{p} \zeta_{k}\left|g_{1}\right|^{2} a_{k}}+\frac{1}{3} \zeta_{k} e^{-a_{k} z_{2} P_{p} \zeta_{k}\left|g_{1}\right|^{2}} \\
+\frac{\left(\frac{(M-1)}{2 M}-\frac{1}{4}\right)}{\sin ^{2} \frac{\pi(M-1)}{M}} \zeta_{k} e^{-a_{k} z_{3} P_{p} \zeta_{k}\left|g_{1}\right|^{2}} \\
=\left(\frac{(M-1)}{2 M}-\frac{1}{6}\right) \zeta_{1} e^{-z_{1} P_{p} \zeta_{1}\left|g_{1}\right|^{2} a_{1}}+\frac{1}{3} \zeta_{1} e^{-a_{1} z_{2} P_{p} \zeta_{1}\left|g_{1}\right|^{2}} \\
+\frac{\left(\frac{(M-1)}{2 M}-\frac{1}{4}\right)}{\sin ^{2} \frac{\pi(M-1)}{M}} \zeta_{1} e^{-a_{1} z_{3} P_{p} \zeta_{1}\left|g_{1}\right|^{2}} .
\end{gathered}
$$

From this expression we can notice that, for a given $\zeta_{1}$ and $\zeta_{k}$, the equality can be satisfied by

$$
\begin{gathered}
\left(\frac{(M-1)}{2 M}-\frac{1}{6}\right) \zeta_{k} e^{-z_{1} P_{p} \zeta_{k}\left|g_{1}\right|^{2} a_{k}}= \\
\left(\frac{(M-1)}{2 M}-\frac{1}{6}\right) \zeta_{1} e^{-z_{1} P_{p} \zeta_{1}\left|g_{1}\right|^{2} a_{1}} \\
\frac{1}{3} \zeta_{k} e^{-a_{k} z_{2} P_{p} \zeta_{k}\left|g_{1}\right|^{2}}=\frac{1}{3} \zeta_{1} e^{-a_{1} z_{2} P_{p} \zeta_{1}\left|g_{1}\right|^{2}} \\
\frac{\left(\frac{(M-1)}{2 M}-\frac{1}{4}\right)}{\sin ^{2} \frac{\pi(M-1)}{M}} \zeta_{k} e^{-a_{k} z_{3} P_{p} \zeta_{k}\left|g_{1}\right|^{2}}= \\
\frac{\left(\frac{(M-1)}{2 M}-\frac{1}{4}\right)}{\sin ^{2} \frac{\pi(M-1)}{M}} \zeta_{1} e^{-a_{1} z_{3} P_{p} \zeta_{1}\left|g_{1}\right|^{2}} .
\end{gathered}
$$

which can be simplified as

$$
\begin{aligned}
e^{-z_{1} P_{p} \zeta_{k}\left|g_{1}\right|^{2} a_{k}} & =\frac{\zeta_{1}}{\zeta_{k}} e^{-z_{1} P_{p} \zeta_{1}\left|g_{1}\right|^{2} a_{1}} . \\
e^{-a_{k} z_{2} P_{p} \zeta_{k}\left|g_{1}\right|^{2}} & =\frac{\zeta_{1}}{\zeta_{k}} e^{-a_{1} z_{2} P_{p} \zeta_{1}\left|g_{1}\right|^{2}} . \\
e^{-a_{k} z_{3} P_{p} \zeta_{k}\left|g_{1}\right|^{2}} & =\frac{\zeta_{1}}{\zeta_{k}} e^{-a_{1} z_{3} P_{p} \zeta_{1}\left|g_{1}\right|^{2}} .
\end{aligned}
$$

By taking, ln, to the two sides in (57), (58) and (59), we can get

$$
\begin{aligned}
& a_{k}=\frac{\zeta_{1} a_{1}}{\zeta_{k}}-\frac{\ln \frac{\zeta_{1}}{\zeta_{k}}}{z_{1} P_{p} \zeta_{k}\left|g_{1}\right|^{2}} . \\
& a_{k}=\frac{\zeta_{1} a_{1}}{\zeta_{k}}-\frac{\ln \frac{\zeta_{1}}{\zeta_{k}}}{z_{2} P_{p} \zeta_{k}\left|g_{1}\right|^{2}} . \\
& a_{k}=\frac{\zeta_{1} a_{1}}{\zeta_{k}}-\frac{\ln \frac{\zeta_{1}}{\zeta_{k}}}{z_{3} P_{p} \zeta_{k}\left|g_{1}\right|^{2}} .
\end{aligned}
$$

In the cases when the users have same path-loss, we can obtain $a_{k}=a_{1}$ from the all three equations (60), (61) and (62). At high SNR values the last three expressions (60), (61) and (62) can be reduced to

$$
a_{k}=\frac{\zeta_{1} a_{1}}{\zeta_{k}} .
$$

Accordingly, we can find

$$
\begin{gathered}
\sum_{k=1}^{K} a_{k}-1=\sum_{k=1}^{K} \frac{\zeta_{1} a_{1}}{\zeta_{k}}-1=0 . \\
a_{1}=\frac{1}{\zeta_{1} \sum_{k=1}^{K} \frac{1}{\zeta_{k}}} .
\end{gathered}
$$

Finally, substituting (65) into (63) we can get the power allocation factor as in Theorem 3. 


\section{APPENDIX D}

Since the average SEP, $P_{e, k}$, depends totally on the received SNR at user $k$, the user who has maximum SEP, $P_{e, \max }$, can be defined as the user who has minimum received SNR, $\gamma_{\min }=\min \left\{\gamma_{1}, \ldots, \gamma_{k}, \ldots, \gamma_{K}\right\}$. Therefore, maximum SEP can be calculated by

$$
P_{e, \max }=\frac{1}{\pi} \int_{0}^{\frac{\pi(M-1)}{M}} \mathcal{M}_{\gamma_{\min }}(z) d \Phi,
$$

where $\mathcal{M}_{\gamma_{\min }}(z)$ is the MGF of the minimum received SNR. In order to find $\mathcal{M}_{\gamma_{\min }}(z)$, we need to find the CDF and/or PDF of $\gamma_{\mathrm{min}}$, which is the distribution of the minimum of dependent random variables. The CDF of $\gamma_{\min }$ can be derived by [51]

$$
F_{\gamma_{\min }}(\bar{\gamma})=1-\operatorname{Pr}\left(\gamma_{1}>\bar{\gamma}, \ldots, \gamma_{k}>\bar{\gamma}, \ldots, \gamma_{K}>\bar{\gamma}\right)
$$

However, the distribution of minimum dependent random variables cannot be obtained in simple closed form expression. On the other hand, some bounds and approximations of this distribution have been provided in several references, for instance in [51, Sections 5.3 and 5.4]. Accordingly, the $\mathrm{CDF}$ of the minimum dependent random variables can be bounded by [51, Section 5.4]

$$
F_{\gamma_{\min }}(\bar{\gamma}) \simeq 1-\prod_{k=1}^{K}\left[1-F_{\gamma_{k}}(\bar{\gamma})\right] .
$$

Now, the MGF of the minimum received SNR can be calculated by

$$
\mathcal{M}_{\gamma_{\text {min }}}(z)=\int_{0}^{\infty} e^{-z \bar{\gamma}} f_{\gamma_{\text {min }}}(\bar{\gamma}) d \bar{\gamma} .
$$

Using integration by parts we can find that

$$
\mathcal{M}_{\gamma_{\text {min }}}(z)=1-z \int_{0}^{\infty} e^{-z \bar{\gamma}}\left(1-F_{\gamma_{\text {min }}}(\bar{\gamma})\right) d \bar{\gamma} .
$$

Substituting (68) into (70) we can get
Applying Gaussian Quadrature rules, the MGF can be written as

$$
\begin{gathered}
\mathcal{M}_{\gamma_{\text {min }}}(z)=1-\sum_{i=1}^{n} \mathrm{H}_{i} \\
\times\left(\prod_{k=1}^{K}\left[1-\frac{\varphi\left(d / p,\left(\bar{\gamma}_{i} / z \alpha_{k} \varrho_{k}\right)^{p}\right)}{\Gamma(d / p)}\right]\right)+R_{i},
\end{gathered}
$$

where $\bar{\gamma}_{i}$ is the $i^{t h}$ zero of the Laguerre polynomials [34]. Substituting (73) into (66), the maximum SEP can be calculated as in (74), where $z=-\frac{\sin ^{2}\left(\frac{\pi}{M}\right)}{\sin ^{2} \Phi}$. Using (45) the max SEP can be written as in (75), which can be simplified as in (76). Now, the Min-Max problem can be formulated as

$$
\min _{a} P_{e, \max }
$$

$$
\text { S.t }: \sum_{k=1}^{K} a_{k}=1, a_{k} \geq 0
$$

which can be expressed using the approximated SEP formula in (76) as

$$
\begin{gathered}
\min _{a}\left(\frac{\Theta}{2 \pi}-\frac{1}{6}\right) \mathcal{M}_{\gamma_{\min }}\left(\sin ^{2}\left(\frac{\pi}{M}\right)\right) \\
+\frac{1}{4} \mathcal{M}_{\gamma_{\min }}\left(\frac{4 \sin ^{2}\left(\frac{\pi}{M}\right)}{3}\right)+\left(\frac{\Theta}{2 \pi}-\frac{1}{4}\right) \mathcal{M}_{\gamma_{\min }}\left(\frac{\sin ^{2}\left(\frac{\pi}{M}\right)}{\sin ^{2} \Theta}\right) \\
\text { S.t }: \sum_{k=1}^{K} a_{k}=1, a_{k} \geq 0
\end{gathered}
$$

and

$$
\begin{gathered}
\min _{a}\left(\frac{\Theta}{\pi}-\frac{1}{6}\right) \\
-c_{1}\left(\sum_{i=1}^{n} \mathrm{H}_{i}\left(\prod_{k=1}^{K}\left[1-\frac{\varphi\left(d / p,\left(\bar{\gamma}_{i} / z_{1} a_{k} P_{p} \zeta_{k} \varrho_{k}\right)^{p}\right)}{\Gamma(d / p)}\right]\right)\right)
\end{gathered}
$$

$\mathcal{M}_{\gamma_{\min }}(z)=1-z \int_{0}^{\infty} e^{-z \bar{\gamma}}\left(1-\left(1-\prod_{k=1}^{K}\left[1-F_{\gamma_{k}}(\bar{\gamma})\right]\right)\right) d \bar{\gamma} . c_{2}\left(\sum_{i=1}^{n} \mathrm{H}_{i}\left(\prod_{k=1}^{K}\left[1-\frac{\varphi\left(d / p,\left(\bar{\gamma}_{i} / z_{2} a_{k} P_{p} \zeta_{k} \varrho_{k}\right)^{p}\right)}{\Gamma(d / p)}\right]\right)\right)$

The CDF of the received SNR can be re-presented as $F_{\gamma_{k}}(\bar{\gamma})=\frac{\varphi\left(d / p,\left(\bar{\gamma} / \alpha_{k} \varrho_{k}\right)^{p}\right)}{\Gamma(d / p)}$, where $\varphi($.$) is the lower$ incomplete Gamma function. Thus,

$$
\begin{gathered}
\mathcal{M}_{\gamma_{\text {min }}}(z)=1-z \int_{0}^{\infty} e^{-z \bar{\gamma}} \\
\times\left(\prod_{k=1}^{K}\left[1-\frac{\varphi\left(d / p,\left(\bar{\gamma} / \alpha_{k} \varrho_{k}\right)^{p}\right)}{\Gamma(d / p)}\right]\right) d \bar{\gamma} .
\end{gathered}
$$

$$
\begin{gathered}
-c_{3}\left(\sum_{i=1}^{n} \mathrm{H}_{i}\left(\prod_{k=1}^{K}\left[1-\frac{\varphi\left(d / p,\left(\bar{\gamma}_{i} / z_{3} a_{k} P_{p} \zeta_{k} \varrho_{k}\right)^{p}\right)}{\Gamma(d / p)}\right]\right)\right) \\
\text { S.t }: \sum_{k=1}^{K} a_{k}=1, a_{k} \geq 0
\end{gathered}
$$

where $c_{1}=\left(\frac{\Theta}{2 \pi}-\frac{1}{6}\right), c_{2}=\frac{1}{4}, c_{3}=\left(\frac{\Theta}{2 \pi}-\frac{1}{4}\right), z_{1}=$ $\sin ^{2}\left(\frac{\pi}{M}\right), z_{2}=\frac{4 \sin ^{2}\left(\frac{\pi}{M}\right)}{3}$ and $z_{3}=\frac{\sin ^{2}\left(\frac{\pi}{M}\right)}{\sin ^{2} \frac{\pi(M-1)}{M}}$. Considering the first-order Laguerre polynomial, we can get (24). 


$$
P_{e, \max }=\frac{1}{\pi} \int_{0}^{\frac{\pi(M-1)}{M}}\left(1-\sum_{i=1}^{n} \mathrm{H}_{i}\left(\prod_{k=1}^{K}\left[1-\frac{\varphi\left(d / p,\left(\bar{\gamma}_{i} / z \alpha_{k} \varrho_{k}\right)^{p}\right)}{\Gamma(d / p)}\right]\right)+R_{i}\right) d \Phi
$$

$$
\begin{aligned}
P_{e, \max } & =\left(\frac{\Theta}{2 \pi}-\frac{1}{6}\right)\left(1-\sum_{i=1}^{n} \mathrm{H}_{i}\left(\prod_{k=1}^{K}\left[1-\frac{\varphi\left(d / p,\left(\bar{\gamma}_{i} / \sin ^{2}\left(\frac{\pi}{M}\right) \alpha_{k} \varrho_{k}\right)^{p}\right)}{\Gamma(d / p)}\right]\right)\right) \\
& +\frac{1}{4}\left(1-\sum_{i=1}^{n} \mathrm{H}_{i}\left(\prod_{k=1}^{K}\left[1-\frac{\varphi\left(d / p,\left(3 \bar{\gamma}_{i} / 4 \sin ^{2}\left(\frac{\pi}{M}\right) \alpha_{k} \varrho_{k}\right)^{p}\right)}{\Gamma(d / p)}\right]\right)\right) \\
+ & \left(\frac{\Theta}{2 \pi}-\frac{1}{4}\right)\left(1-\sum_{i=1}^{n} \mathrm{H}_{i}\left(\prod_{k=1}^{K}\left[1-\frac{\varphi\left(d / p,\left(\bar{\gamma}_{i} \sin ^{2} \Theta / \sin ^{2}\left(\frac{\pi}{M}\right) \alpha_{k} \varrho_{k}\right)^{p}\right)}{\Gamma(d / p)}\right]\right)\right) .
\end{aligned}
$$

$$
\begin{aligned}
P_{e, \max }= & \left(\frac{\Theta}{\pi}-\frac{1}{6}\right)-\left(\frac{\Theta}{2 \pi}-\frac{1}{6}\right)\left(\sum_{i=1}^{n} \mathrm{H}_{i}\left(\prod_{k=1}^{K}\left[1-\frac{\varphi\left(d / p,\left(\bar{\gamma}_{i} / z \alpha_{k} \varrho_{k}\right)^{p}\right)}{\Gamma(d / p)}\right]\right)\right) \\
& -\frac{1}{4}\left(\sum_{i=1}^{n} \mathrm{H}_{i}\left(\prod_{k=1}^{K}\left[1-\frac{\varphi\left(d / p,\left(\bar{\gamma}_{i} / z \alpha_{k} \varrho_{k}\right)^{p}\right)}{\Gamma(d / p)}\right]\right)\right) \\
& -\left(\frac{\Theta}{2 \pi}-\frac{1}{4}\right)\left(\sum_{i=1}^{n} \mathrm{H}_{i}\left(\prod_{k=1}^{K}\left[1-\frac{\varphi\left(d / p,\left(\bar{\gamma}_{i} / z \alpha_{k} \varrho_{k}\right)^{p}\right)}{\Gamma(d / p)}\right]\right)\right) .
\end{aligned}
$$

\section{REFERENCES}

[1] A. Salem and C. Masouros, "ON the ERror Probability of INTERFERENCE EXPLOITATION PRECODING WITH POWER ALLOCATIOn," in Proc. IEEE Wireless Commun. Netw. Conf. (WCNC), 2020.

[2] M. S. John G. Proakis, Digital Communications, Fifth Edition. MCGRAW-HILL, NY USA, 2008.

[3] C. B. P. Howard Huang And S. Venkatesan, MIMO Communication for cellular Networks. SPRINGER, 2008.

[4] Y. Wu, C. XiaO, X. GaO, J. D. Matyjas, AND Z. Ding, "Linear PRECODER DESIGN FOR MIMO INTERFERENCE CHANNELS WITH FINITE-ALPHABET SIGNALING," IEEE Transactions on Communications, VOL. 61, NO. 9, PP. 3766-3780, SEPTEMBER 2013.

[5] A. SAlEm AND K. A. HAMdi, "Wireless POWER TRANSFER IN MULTI-PAIR TWO-WAY AF RELAYING NETWORKS," IEEE Transactions on Communications, VOL. 64, NO. 11, PP. 4578-4591, NOV 2016.

[6] W. Wu, K. Wang, W. Zeng, Z. Ding, and C. Xiao, "CoOPERATIVE MULTI-CELL MIMO DOWNLINK PRECODING WITH FINITE-ALPHABET INPUTS," IEEE Transactions on Communications, VOL. 63, NO. 3, PP. 766-779, MARCH 2015.

[7] M. COSTA, "Writing ON DIRTY PAPER (CORRESP.)," IEEE Transactions on Information Theory, VOL. 29, NO. 3, PP. 439-441, MAY 1983.

[8] C. Masouros, M. Sellathurai, and T. Ratnarajah, "MaXIMIZING ENERGY EFFICIENCY IN THE VECTOR PRECODED MUMISO DOWNLINK BY SELECTIVE PERTURBATION," IEEE Transactions on Wireless Communications, vOL. 13, NO. 9, PP. 4974-4984, SEP. 2014.

[9] A. Garcia-Rodriguez and C. Masouros, "Power-efFicient TOMLINSON-HARASHIMA PRECODING FOR THE DOWNLINK OF MULTI-USER MISO SYSTEMS," IEEE Transactions on Communications, VOL. 62, NO. 6, PP. 1884-1896, JUNE 2014.
[10] A. Li and C. Masouros, "INTERfEREnCE EXPloitation PRECODING MADE PRACTICAL: OPTIMAL CLOSED-FORM SOLUTIONS FOR PSK MODULATIONS," IEEE Transactions on Wireless Communications, PP. 1-1, 2018.

[11] T. Haustein, C. von Helmolt, E. Jorswieck, V. JungNICKEL, AND V. POHL, "PERFORMANCE OF MIMO SYSTEMS WITH CHANNEL INVERSION," IN Vehicular Technology Conference. IEEE 55th Vehicular Technology Conference. VTC Spring 2002 (Cat. No.02CH37367), vOL. 1, MAY 2002, PP. 35-39 vOL.1.

[12] C. B. Peel, B. M. Hochwald, and A. L. Swindlehurst, "A VECTOR-PERTURBATION TECHNIQUE FOR NEAR-CAPACITY MULTIANTENNA MULTIUSER COMMUNICATION-PART I: CHANNEL INVERSION AND REGULARIZATION," IEEE Transactions on Communications, VOL. 53, NO. 1, PP. 195-202, JAN 2005.

[13] A. Wiesel, Y. C. Eldar, AND S. Shamai, "LiNEAR PRECODinG VIA CONIC OPTIMIZATION FOR FIXED MIMO RECEIVERS," IEEE Transactions on Signal Processing, vOL. 54, NO. 1, PP. 161-176, JAN 2006.

[14] M. F. Hanif, L. Tran, A. TÖlli, and M. Juntti, "ComputaTIONALLY EFFICIENT ROBUST BEAMFORMING FOR SINR BALANCING IN MULTICELL DOWNLINK WITH APPLICATIONS TO LARGE ANTENNA ARRAY SYSTEMS," IEEE Transactions on Communications, VOL. 62, NO. 6, PP. 1908-1920, JUNE 2014.

[15] M. Schubert and H. Boche, "Solution of the MUlTIUSER DOWNLINK BEAMFORMING PROBLEM WITH INDIVIDUAL SINR CONSTRAINTS," IEEE Transactions on Vehicular Technology, VOL. 53, NO. 1, PP. 18-28, JAN 2004.

[16] N. D. Sidiropoulos, T. N. Davidson, And Zhi-Quan Luo, "TRANSMIT BEAMFORMING FOR PHYSICAL-LAYER MULTICASTING," IEEE Transactions on Signal Processing, VOL. 54, NO. 6, PP. 2239-2251, JUNE 2006.

[17] A. Li, D. Spano, J. Krivochiza, S. Domouchtsidis, C. G. Tsinos, C. Masouros, S. Chatzinotas, Y. Li, B. Vucetic, AND B. OTTERSTEN, "A TUTORIAL ON INTERFERENCE EXPLOITATION VIA SYMBOL-LEVEL PRECODING: OVERVIEW, STATE-OF- 
THE-ART AND FUtURE DiReCTIONS," IEEE Communications Surveys Tutorials, VOL. 22, NO. 2, PP. 796-839, 2020.

[18] C. Masouros and E. Alsusa, "Dynamic linear Precoding FOR THE EXPLOITATION OF KNOWN INTERFERENCE IN MIMO BROADCAST SYSTEMS," IEEE Transactions on Wireless Communications, VOL. 8, NO. 3, PP. 1396-1404, MARCH 2009.

[19] C. MASOURos AND G. ZHENG, "EXPLOITING KNOWN INTERFERENCE AS GREEN SIGNAL POWER FOR DOWNLINK BEAMFORMING OPTIMIZATION," IEEE Transactions on Signal Processing, vOL. 63, NO. 14, PP. 3628-3640, JULY 2015.

[20] S. Timotheou, G. Zheng, C. Masouros, and I. Krikidis, "EXPLOITING CONSTRUCTIVE INTERFERENCE FOR SIMULTANEOUS WIRELESS INFORMATION AND POWER TRANSFER IN MULTIUSER DOWNLINK SYSTEMS," IEEE Journal on Selected Areas in Communications, vOL. 34, NO. 5, PP. 1772-1784, MAY 2016.

[21] M. R. A. Khandaker, C. Masouros, And K. K. Wong, "CONSTRUCTIVE INTERFERENCE BASED SECURE PRECODING: A NEW DIMENSION IN PHYSICAL LAYER SECURITY," IEEE Transactions on Information Forensics and Security, VOL. 13, NO. 9, PP. 22562268, SEPT 2018

[22] C. Masouros, M. Sellathurai, and T. Ratnarajah, "VecTOR PERTURBATION BASED ON SYMBOL SCALING FOR LIMITED FEEDBACK MISo DOWNLINKS," IEEE Transactions on Signal Processing, VOL. 62, NO. 3, PP. 562-571, FEB 2014.

[23] A. HaqiqATneJad, F. KAYhan, AND B. OtTersten, "SymbolLEVEL PRECODING DESIGN BASED ON DISTANCE PRESERVING CONSTRUCTIVE INTERFERENCE REGIONS," IEEE Transactions on Signal Processing, vOL. 66, NO. 22, PP. 5817-5832, Nov 2018.

[24] — - "CONSTRUCTIVE INTERFERENCE FOR GENERIC CONSTELLATIONS," IEEE Signal Processing Letters, vOL. 25, NO. 4, PP. 586-590, APRIL 2018.

[25] A. Salem, C. Masouros, and K. Wong, "Sum Rate and FAIRNESS ANALYSIS FOR THE MU-MIMO DOWNLINK UNDER PSK SIGNALLING: INTERFERENCE SUPPRESSION VS EXPLOITATION," IEEE Transactions on Communications, PP. 1-1, 2019.

[26] A. Salem, C. Masouros, and B. Clerckx, "Rate SplitTing With Finite Constellations: The Benefits of INTERFERENCE EXPLOITATION VS SUPPRESSION," arXiv e-prints, P. ARXIV: 1907.08457, JUL 2019.

[27] A. Salem and C. Masouros, "Rate splitting approach UNDER PSK SIGNALING USING CONSTRUCTIVE INTERFERENCE PRECODING TECHNIQUE," IN 2019 IEEE Wireless Communications and Networking Conference (WCNC), APRIL 2019, PP. 1-6.

[28] ——, "ON THE FINITE CONSTELLATION SUM RATES FOR ZF AND CI PRECODING," IN 2019 IEEE Wireless Communications and Networking Conference (WCNC), APRIL 2019, PP. 1-6.

[29] E. Biglieri, G. CAire, and G. TARicCo, "Limiting PERforMANCE OF BLOCK-FADING CHANNELS WITH MULTIPLE ANTENNAS," IEEE Transactions on Information Theory, VOL. 47, NO. 4, PP. 1273-1289, MAY 2001.

[30] C. R. RAO, Linear Statistical Inference and its Applications: Second Editon. 1973 JoHN WILEY AND Sons, 13 APRIL 1973

[31] A. MeuCCI, Risk and Asset Allocation. SPRINGER, 2005.

[32] R. J. MuIRHEAD, Aspects of Multivariate Statistical Theory, 1982.

[33] M. L. EATON, Chapter 8: The Wishart Distribution, SER. Lecture notes-Monograph Series. Beachwood, Ohio, USA: Institute of Mathematical Statistics, 2007, vol. Volume 53, PP. 302-333. [ONLINE]. AVAilable: HTtPs://DOI. ORG/10.1214/LNMS/1196285114

[34] M. Abramowitz And I. A. Stegun, Handbook of Mathematical Functions With Formulas, Graphs, and Mathematical Tabl, WASHINGTON,D.C.: U.S. DEPT. COMMERCE, 1972.

[38] P. V. AMAdori and C. Masouros, "Large scale antenna SELECTION AND PRECODING FOR INTERFERENCE EXPLOITA-
[35] M. K. Simon And M. S. Alouini, Digital Communication over Fading Channels. JOHN WILEY AND SONS, 2000.

[36] M. R. Mckay, A. Zanella, I. B. Collings, and M. Chiani, "ERROR PROBABILITY AND SINR ANALYSIS OF OPTIMUM COMBINING IN RICIAN FADING," IEEE Transactions on Communications, vOL. 57, NO. 3, PP. 676-687, MARCH 2009.

[37] C. MASOUROS, "CORRELATION ROTATION LINEAR PRECODING FOR MIMO BROADCAST COMMUNICATIONS," IEEE Transactions on Signal Processing, vOL. 59, NO. 1, PP. 252-262, JAN 2011. TION," IEEE Transactions on Communications, VOL. 65, NO. 10, PP. 4529-4542, ОСт 2017.

[39] A. T. Toyserkani, E. G. Strom, and A. Svensson, "An ANALYTICAL APPROXIMATION TO THE BLOCK ERROR RATE IN NAKAGAMI-M NON-SELECTIVE BLOCK FADING CHANNELS," IEEE Transactions on Wireless Communications, vOL. 9, NO. 5, PP. 15431546, MAY 2010.

[40] R. Eaves and A. Levesque, "Probability of Block error FOR VERY SLOW RAYLEIGH FADING IN GAUSSIAN NOISE," IEEE Transactions on Communications, vOL. 25, NO. 3, PP. 368-374, MARCH 1977.

[41] F. Adachi and T. Matsumoto, "Double symbol error Rate AND BLOCK ERROR RATE OF MDPSK," Electronics Letters, vOL. 27, NO. 17, PP. 1571-1573, AUg 1991

[42] A. Seyoum and N. C. Beaulieu, "Semianalytical simuLATION FOR EVALUATION OF BLOCK-ERROR RATES ON FADING CHANNELS," IEEE Transactions on Communications, VOL. 46, NO. 7, PP. 916-920, JULY 1998.

[43] M. Ruiz-Garcia, J. M. Romero-Jerez, C. Tellez-Labao, AND A. Diaz-Estrella, "Average block ERror probabilITY OF MULTICELL CDMA PACKET NETWORKS WITH FAST POWER CONTROL UNDER MULTIPATH FADING," IEEE COmmunications Letters, VOL. 6, NO. 12, PP. 538-540, DEC 2002.

[44] Jianhua Lu, K. B. Letaief, J. C. . Chuang, and M. L. Liou, "M-PSK AND M-QAM BER COMPUTATION USING SIGNAL-SPACE CONCEPTS," IEEE Transactions on Communications, vOL. 47, NO. 2, PP. 181-184, FEB 1999.

[45] M. ChiAni, "ERror PROBABility For Block CODES OVER CHANNELS WITH BLOCK INTERFERENCE," IEEE Transactions on Information Theory, vOL. 44, NO. 7, PP. 2998-3008, Nov 1998.

[46] A. Garcia-Rodriguez and C. Masouros, "Exploiting the INCREASING CORRELATION OF SPACE CONSTRAINED MASSIVE MIMO FOR CSI RELAXATION," IEEE Transactions on Communications, VOL. 64, NO. 4, PP. 1572-1587, APRIL 2016.

[47] G. Auer, V. Giannini, I. Godor, P. Skillermark, M. OlsSon, M. A. ImRan, D. SABella, M. J. GonZalez, C. Desset, AND O. Blume, "Cellular ENERGY EFFICIENCY EVAluation FRAMEWORK," IN 2011 IEEE 73rd Vehicular Technology Conference (VTC Spring), MAY 2011, PP. 1-6.

[48] H. Kim, C. Chae, G. DE Veciana, and R. W. Heath, "A crossLAYER APPROACH TO ENERGY EFFICIENCY FOR ADAPTIVE MIMO SYSTEMS EXPLOITING SPARE CAPACITY," IEEE Transactions on Wireless Communications, VOL. 8, NO. 8, PP. 4264-4275, AUGUST 2009.

[49] M. Chiani, D. Dardari, AND M. K. Simon, "New exponenTIAL BOUNDS AND APPROXIMATIONS FOR THE COMPUTATION OF ERROR PROBABILITY IN FADING CHANNELS," IEEE Transactions on Wireless Communications, VOL. 2, NO. 4, PP. 840-845, JULY 2003.

[50] S. Boyd and L. Vandenberghe, Convex Optimization. CAMBRIDGE, UK: CAMBRIDGE UNIVERSITY. PRESS, 2004.

[51] H. DAvid., Order Statistics, 1970:John Wiley AND Sons. 